\title{
Induced fermionic vacuum polarization in a de Sitter spacetime with a compactified cosmic string
}

\author{
E. A. F. Bragança $\oplus^{1, \dagger}$ E. R. Bezerra de Mello, ${ }^{2, \dagger}$ and A. Mohammadi $\odot^{1, *}$ \\ ${ }^{1}$ Departamento de Física, Universidade Federal de Pernambuco, 52171-900 Recife, PE, Brazil \\ ${ }^{2}$ Departamento de Física, Universidade Federal da Paraíba \\ 58.059-970, Caixa Postal 5.008 João Pessoa, PB, Brazil
}

(Received 16 October 2019; accepted 10 February 2020; published 26 February 2020)

\begin{abstract}
We study the fermionic condensate (FC) and the vacuum expectation value (VEV) of the energymomentum tensor for a massive spinor field in the de Sitter (dS) spacetime including an ideal cosmic string. In addition, spatial dimension along the string is compactified to a circle of length $L$. The fermionic field is assumed to obey quasiperiodic condition along the $z$-axis. There are also magnetic fluxes running along the cosmic string and enclosed by the compact dimension. Both, the FC and the VEV of the energy-momentum tensor, are decomposed into two parts: one induced by the cosmic string in dS spacetime considering the absence of the compactification, and another one induced by the compactification. In particular, we show that the FC vanishes for a massless fermionic field.
\end{abstract}

DOI: $10.1103 /$ PhysRevD.101.045019

\section{INTRODUCTION}

It is well known that in the early Universe different types of topological defects may have been formed due to the series of phase transitions [1] which among them, cosmic strings have been extensively studied in the literature. Observations of the cosmic microwave background have ruled cosmic strings out as the main source of the primordial density fluctuations, but several other interesting physical effects can be associated with this topological defect like emission of gravitational waves, generation of high-energy cosmic rays and doubling images of distant objects [2-4]. Furthermore, a variant in the mechanism of formation for cosmic strings has been proposed in the context of brane inflation [5-7] leading to a renewed interest in the research on this object. The topological defects can also form in condensed matter systems due to symmetry breaking phase transitions. The conical space can appear as an effective background geometry in certain condensed matter systems such as nanotubes, superfluids, superconductors, crystals, liquid crystals, and quantum liquids [8,9].

The geometry of the spacetime associated with an infinitely long and straight cosmic string is characterized

\footnotetext{
${ }^{*}$ Corresponding author. azadeh.mohammadi@df.ufpe.br

tbraganca@df.ufpe.br

*emello@fisica.ufpb.br
}

Published by the American Physical Society under the terms of the Creative Commons Attribution 4.0 International license. Further distribution of this work must maintain attribution to the author(s) and the published article's title, journal citation, and DOI. Funded by SCOAP ${ }^{3}$. by a planar angle deficit on the two-surface orthogonal to the string. Besides that, the spacetime is locally flat except on the top of the string where a delta shaped curvature tensor is present. Cosmic strings were first introduced in the literature as being created by a Dirac-delta type distribution of energy and an axial stress, however it can also be described in the context of a classical field theory which the energy-momentum tensor associated with the vortex configuration of the Maxwell-Higgs system [10] couples to the Einstein equations. Garfinkle [11] and Linet [12] have studied this coupled system and have shown that a planar angle deficit arises on the two-surface perpendicular to the string, as well as a magnetic flux running along to its core. One of the most remarkable features of this spacetime is the fact that fields are sensitive to its global conical structure which can cause interesting phenomena.

It is well known that geometry and topology play important roles in many physical problems with implications from subnuclear to cosmological scales. In the context of the quantum field theory, the vacuum properties are influenced by both geometrical and topological aspects of the background spacetime. In the present paper, we intend to investigate the combined effects of the geometry and topology on the fermionic condensate and the VEV of the energy-momentum tensor associated with a massive fermion field. We take the de Sitter spacetime as the background geometry and that the topological effects are induced by the presence of a cosmic string and the compactification of the spatial dimension along the string.

The dS spacetime is the curved spacetime most analyzed in the context of quantum field theory and cosmology. Considering the presence of a positive cosmological 
constant, the dS spacetime is a maximally symmetric solution of Einstein equations. As a consequence of this high degree of symmetry, several numbers of physical problems can be exactly solved in this spacetime. In addition, the importance of this background has increased after the advent of the Universe's expansion in its early stages from an inflationary scenario. In this scenario the geometry of the Universe can be approximated by a portion of the dS spacetime and due to this fact some problems in the standard cosmology are naturally solved. Besides that, during the inflationary epoch, fluctuations in the inflaton field have generated inhomogeneities that play an important role in the generation of large scale structures in the Universe [13].

The type of topological effect we shall consider here is induced by a planar angle deficit due the presence of a cosmic string. The conical structure of the spacetime associated with this topological defect modifies the vacuum fluctuations associated to quantum fields. In this way, the vacuum expectation values (VEVs) of the energy-momentum tensor associated with scalar [14-18] and fermion [19-21] fields present a nonzero value. Additional contributions to the above VEVs associated with charged quantum fields are induced when is considered the presence of a magnetic flux running along the string [22-26]. Vacuum current densities are also induced by the magnetic fluxes and it has been shown that the azimuthal induced current density arises if the ratio of the magnetic flux by the quantum one has a nonzero fractional part [27,28]. In addition, induced current densities in a higher-dimensional cosmic string spacetime [29] and in a $(2+1)$-dimensional cosmic string spacetime with a circular boundary [30] in the presence of a magnetic flux were also studied. In the aforementioned analysis the cosmic string had no inner structure, i.e., it is considered as an ideal linear object. The inner structure of the cosmic string has been taken into consideration in Refs. [31,32] for the scalar and fermionic currents and also for the energy-momentum tensor in [33].

The compactification along the cosmic string axis can also induce a topological effect in the system. Most high-energy theories of fundamental physics, like supergravity and superstring theories, have this feature in common. An interesting application of a theoretical model with a compact dimension appeared in the context of nanophysics. In cylindrical and toroidal carbon nanotubes, the background of the corresponding field theory presents compact dimensions [34]. In the context of quantum field theory, the imposition of periodicity conditions on the field operator along compact dimension also alters the VEVs of physical observables. In $[35,36]$ the fermionic current in the presence of an arbitrary number of compactified spatial dimensions and a constant gauge was investigated. The VEV of the induced fermionic current and the energy-momentum tensor considering a compactified cosmic string spacetime in the presence of a magnetic flux running through the string was studied in [37,38]. Moreover, for a charged scalar field, the VEVs of the induced bosonic current and energy-momentum tensor in a higher dimensional compactified cosmic string spacetime was considered in $[39,40]$, respectively. For Schwarzschild spacetime equipped by a cosmic string, the vacuum polarization was investigated in [41,42]. For the dS spacetime, the vacuum polarization induced by a cosmic string for a scalar field [43] and a fermion field [44], and the calculation of the vacuum fermionic current [45] have been developed. In addition, similar analysis induced by a cosmic string in anti-dS spacetime have also been considered for massive scalar [46] and fermion fields [47], and for a scalar field with a compactified extra dimension [48]. With the intention of developing a further analysis, here we plan to investigate the fermionic vacuum polarization and the VEV of the energy-momentum tensor in dS spacetime, considering the presence of a compactified cosmic string. In this way, this present analysis is as general as possible. However, in the limit where the size of the compactification goes to infinity one recovers the result in the absence of the compactification.

The paper is organized as follows. In Sec. II we describe the background geometry and the complete set of normalized positive- and negative-energy fermionic mode function which obey a quasiperiodic boundary condition with an arbitrary phase along the $z$-axis. We also assume the presence of a constant gauge field. In Sec. III, by using the modesummation method we develop the analysis of the FC which is decomposed into two contributions: the first one corresponds to the geometry of a cosmic string in dS spacetime with no compactification and the second one induced by the compactification of the spatial dimension along the string. In Sec. IV we develop the calculation of the VEV of the energymomentum tensor, proving the same decomposition. In Sec. V we study some properties of the results obtained in the previous section and discuss some limiting cases. Our conclusions are summarized in Sec. VI. Throughout the paper we use natural units in which $G=\hbar=c=1$.

\section{BACKGROUND GEOMETRY AND FERMIONIC MODES}

The line element describing a cosmic string along the $z$-axis in dS spacetime is given by

$d s^{2}=g_{\mu \nu} d x^{\mu} d x^{\nu}=d t^{2}-e^{2 t / \alpha}\left(d r^{2}+r^{2} d \phi^{2}+d z^{2}\right)$,

in cylindrical coordinates with non-negative $r, t \in$ $(-\infty,+\infty)$ and $\phi \in[0,2 \pi / q]$ where the presence of the cosmic string is codified by the parameter $q>1$. The parameter $\alpha$ appearing in the line element above is related to the Ricci scalar $R$ and the cosmological constant $\Lambda$ as $R=12 \alpha^{-2}$ and $\Lambda=3 \alpha^{-2}$. Making use of the conformal time $\eta$, which is defined as [43]

$$
\eta=\alpha e^{-t / \alpha}, \quad \eta \in[0, \infty],
$$


we can write the line element (2.1) in the following form

$$
d s^{2}=(\alpha / \eta)^{2}\left(d \eta^{2}-d r^{2}-r^{2} d \phi^{2}-d z^{2}\right) .
$$

Besides that, the direction along the $z$-axis is compactified to a circle with length $L$, meaning $z \in[0, L]$, and we assume that along this compact direction the fermionic field obeys the following quasiperiodicity condition

$$
\psi(t, r, \phi, z+L)=e^{2 \pi i \beta} \psi(t, r, \phi, z) .
$$

The parameter $\beta$ in the above expression is defined as a constant in the interval $[0,1]$. We have two special cases for this parameter: for $\beta=0$ we have a peridioc boundary condition, while for $\beta=1 / 2$ we have an antiperiodic boundary condition. These values correspond to untwisted and twisted fields, respectively.

The dynamics of a massive spinor field in the above curved spacetime with a magnetic flux running along the string is governed by the Dirac equation in the following form

$$
i \gamma^{\mu} \mathcal{D}_{\mu} \psi-m \psi=0, \quad \mathcal{D}_{\mu}=\partial_{\mu}+i e A_{\mu}+\Gamma_{\mu},
$$

knowing that $\Gamma_{\mu}$ and $A_{\mu}$ are the spin connection and a four vector potential, respectively. Note that the physical components of the vector potential, $A_{\phi}$ and $A_{z}$, are related to the covariant components by $A_{\phi}=-A_{2} / r$ and $A_{z}=-A_{3}$ in the Dirac equation (2.5). To obtain the fermionic condensate as well as the vacuum expectation value of the energymomentum tensor one needs a complete set of fermionic modes. The positive- and negative-energy fermionic modes in this spacetime background were obtained in [45] which are

$$
\begin{aligned}
\psi_{\sigma}^{( \pm)}(x)= & c_{\sigma}^{( \pm)} \eta^{2} e^{i q(j+a) \phi+i k z} \\
& \times\left(\begin{array}{c}
H_{1 / 2-i m \alpha}^{\left(\lambda_{ \pm}\right)}(\gamma \eta) J_{\beta_{1}}(p r) e^{-i q \phi / 2} \\
\frac{i s p \epsilon_{j}}{\gamma+s k} H_{1 / 2-i m \alpha}^{\left(\lambda_{ \pm}\right)}(\gamma \eta) J_{\beta_{2}}(p r) e^{i q \phi / 2} \\
-i s H_{-1 / 2-i m \alpha}^{\left(\lambda_{ \pm}\right)}(\gamma \eta) J_{\beta_{1}}(p r) e^{-i q \phi / 2} \\
\frac{p \epsilon_{j}}{\gamma+s k} H_{-1 / 2-i m \alpha}^{\left(\lambda_{ \pm}\right)}(\gamma \eta) J_{\beta_{2}}(p r) e^{i q \phi / 2}
\end{array}\right),
\end{aligned}
$$

where the gauge transformation $A_{\mu} \rightarrow A_{\mu}+\partial_{\mu} \Lambda, \psi \rightarrow$ $e^{-i e \Lambda} \psi$ with $\Lambda=-A_{\mu} x^{\mu}$ leading to the new Dirac equation, $\left(i \gamma^{\mu} \nabla_{\mu}-m\right) \psi=0$, was used. The above fermionic modes are specified by the set $\sigma=(p, k, j, s)$ of quantum numbers. In the above expression, $\lambda_{+}=1, \lambda_{-}=2$, $j= \pm 1 / 2, \pm 3 / 2, \ldots$, and $s= \pm 1$. In addition, $J_{\nu}(x)$ and $H_{\nu}^{(1,2)}(x)$ are Bessel and Hankel functions [49], respectively, and $\gamma=\sqrt{k^{2}+p^{2}}$ where $0 \leq p<\infty$. Moreover, in the Eq. (2.6) we have

$$
\begin{aligned}
\beta_{1} & =q|j+a|-\epsilon_{j} / 2, \quad \beta_{2}=q|j+a|+\epsilon_{j} / 2, \\
\left|c_{\sigma}^{( \pm)}\right|^{2} & =\frac{q p e^{ \pm m \alpha \pi}}{16 L \alpha^{3}}(\gamma+s k),
\end{aligned}
$$

with $k=k_{l}=2 \pi(l+\tilde{\beta}) / L, l=0, \pm 1, \pm 2, \ldots$ and

$$
a=\frac{\Phi_{\phi}}{\Phi_{0}} \quad \text { and } \quad \tilde{\beta}=\beta-\frac{\Phi_{z}}{\Phi_{0}},
$$

where $\Phi_{0}=2 \pi / e$ is the quantum flux while $\Phi_{\varphi}$ and $\Phi_{z}$ are the magnetic fluxes in the azimuthal and axial directions, respectively. Besides that, $\epsilon_{j}=1$ for $j>-a$ and $\epsilon_{j}=-1$ for $j<-a$.

One can write the parameter $a$ in the form

$$
a=n_{0}+a_{0}, \quad\left|a_{0}\right|<1 / 2,
$$

with $n_{0}$ being an integer number. It is easy to see that the VEVs of the physical observables are given in terms of $a_{0}$ solely, by simply a shift like $j+n_{0} \rightarrow j$.

In [45], we studied the vacuum fermionic currents in the same geometry, a compactified cosmic string in the background of dS spacetime assuming that the field is prepared in the Bunch-Davies vacuum state. In the continuation of the work, we investigate the fermionic condensate and the renormalized vacuum expectation value of the energymomentum tensor in the present paper.

\section{FERMIONIC CONDENSATE}

In this section we develop the analysis of the FC, which is defined by the VEV

$$
\langle 0|\bar{\psi} \psi| 0\rangle \equiv\langle\bar{\psi} \psi\rangle=\sum_{\sigma} \bar{\psi}_{\sigma}^{(-)} \psi_{\sigma}^{(-)}
$$

with $|0\rangle$ representing the vacuum state and $\bar{\psi}=\psi^{\dagger} \gamma^{0}$ being the Dirac adjoint. By using the mode functions (2.6) one can write the FC as

$$
\begin{aligned}
\langle\bar{\psi} \gamma\rangle= & \frac{q \eta^{4} e^{-m \alpha \pi}}{16 L \alpha^{3}} \sum_{\sigma} p(\gamma+s k)\left[J_{\beta_{1}}^{2}(p r)+\frac{p^{2}}{(\gamma+s k)^{2}} J_{\beta_{2}}^{2}(p r)\right] \\
& \times\left[\left|H_{1 / 2-i m \alpha}^{(2)}(\gamma \eta)\right|^{2}-\left|H_{-1 / 2-i m \alpha}^{(2)}(\gamma \eta)\right|^{2}\right]
\end{aligned}
$$

using the compact notation for the sum over all independent quantum numbers as

$$
\sum_{\sigma}=\int_{0}^{\infty} d p \sum_{l=-\infty}^{+\infty} \sum_{s= \pm 1} \sum_{j= \pm 1 / 2, \pm 3 / 2, \ldots} .
$$

Making use of (A1) and evaluating the summation over $s$ result in 


$$
\begin{aligned}
\langle\bar{\psi} \psi\rangle= & \frac{q \eta^{4}}{2 \pi^{2} L \alpha^{3}} \int_{0}^{\infty} d p \sum_{l=-\infty}^{+\infty} \sum_{j} \gamma p\left[J_{\beta_{1}}^{2}(p r)+J_{\beta_{2}}^{2}(p r)\right] \\
& \times\left[\left|K_{1 / 2-i m \alpha}(i \gamma \eta)\right|^{2}-\left|K_{1 / 2+i m \alpha}(i \gamma \eta)\right|^{2}\right] .
\end{aligned}
$$

By means of the relation (A2) we obtain

$$
\begin{aligned}
\langle\bar{\psi} \psi\rangle= & -\frac{i q \eta^{4}}{2 \pi^{2} \alpha^{3} L}\left(\partial_{\eta}+\frac{1-2 i m \alpha}{\eta}\right) \\
& \times \int_{0}^{\infty} d p p \sum_{j}\left[J_{\beta_{1}}^{2}(p r)+J_{\beta_{2}}^{2}(p r)\right] \\
& \times \sum_{l=-\infty}^{\infty} K_{1 / 2-i m \alpha}(i \gamma \eta) K_{1 / 2-i m \alpha}(-i \gamma \eta),
\end{aligned}
$$

being $\gamma=\gamma_{l}=\sqrt{p^{2}+(2 \pi(l+\tilde{\beta}) / L)^{2}}$ and $K_{\nu}(z)$ the Macdonald function [49]. The summation over $l$ can be evaluated with the help of the Abel-Plana formula in the form [50-52]

$$
\begin{aligned}
\frac{2 \pi}{L} \sum_{l=-\infty}^{+\infty} g\left(k_{l}\right) f\left(\left|k_{l}\right|\right)= & \int_{0}^{\infty} d u[g(u)+g(-u)] f(u) \\
& +i \int_{0}^{\infty} d u[f(i u)-f(-i u)] \\
& \times \sum_{\chi= \pm 1} \frac{g(i \chi u)}{e^{L u+2 \pi i \chi \tilde{\beta}}-1},
\end{aligned}
$$

where one can decompose the FC in the following way

$$
\langle\bar{\psi} \psi\rangle=\langle\bar{\psi} \psi\rangle_{s}^{d S}+\langle\bar{\psi} \psi\rangle_{c} .
$$

The first term in the right-hand side of the above equation is the contribution for the FC due to the curvature of the dS spacetime in the cosmic string background with no compactification while the second one is the contribution induced by the compactification along the z-axis. Obviously, the second term should vanish in the limit $L \rightarrow \infty$.

By choosing $g(u)=1$ and

$$
f(u)=K_{1 / 2-i m \alpha}(i \gamma \eta) K_{1 / 2-i m \alpha}(-i \gamma \eta),
$$

the first term in the Eq. (3.6) gives

$$
\begin{aligned}
\langle\bar{\psi} \psi\rangle_{s}^{d S}= & -\frac{i q \eta^{4}}{2 \pi^{3} \alpha^{3}}\left(\partial_{\eta}+\frac{1-2 i m \alpha}{\eta}\right) \\
& \times \int_{0}^{\infty} d p p \sum_{j}\left[J_{\beta_{1}}^{2}(p r)+J_{\beta_{2}}^{2}(p r)\right] \\
& \times \int_{0}^{\infty} d k K_{1 / 2-i m \alpha}(i \gamma \eta) K_{1 / 2-i m \alpha}(-i \gamma \eta) .
\end{aligned}
$$

Using the integral representation (A3) as well as the relation (A10), the integral over $k$ and $p$ can be done directly which gives

$$
\begin{aligned}
\langle\bar{\psi} \psi\rangle_{s}^{d S}= & -\frac{i q}{2^{9 / 2} \pi^{5 / 2} \alpha^{3}}\left(\eta \frac{\partial}{\partial \eta}+1-2 i m \alpha\right) \\
& \times \int_{0}^{\infty} d y \frac{\cosh [(1-2 i m \alpha) y]}{\sinh ^{3} y} \\
& \times \int_{0}^{\infty} \frac{d u}{u^{5 / 2}} e^{-1 /(2 u)-r^{2} /\left(4 u \eta^{2} \sinh ^{2} y\right)} \\
& \times \mathcal{J}\left(q, a_{0}, \frac{r^{2}}{4 u \eta^{2} \sinh ^{2} y}\right),
\end{aligned}
$$

with the notation

$$
\mathcal{J}\left(q, a_{0}, w\right)=\sum_{j}\left[I_{\beta_{1}}(w)+I_{\beta_{2}}(w)\right]
$$

Defining a new variable $x \equiv r^{2} /\left(4 u \eta^{2} \sinh ^{2} y\right)$ the integral over $y$ can be evaluated directly and we obtain

$$
\begin{aligned}
\langle\bar{\psi} \psi\rangle_{s}^{d S}= & -\frac{i q \eta^{3}}{(2 \pi)^{5 / 2}(\alpha r)^{3}}\left(\eta \frac{\partial}{\partial \eta}+1-2 i m \alpha\right) \\
& \times \int_{0}^{\infty} d x x^{1 / 2} e^{x\left(\eta^{2} / r^{2}-1\right)} \\
& \times K_{1 / 2-i m \alpha}\left(x \eta^{2} / r^{2}\right) \mathcal{J}\left(q, a_{0}, x\right),
\end{aligned}
$$

Now, considering the formula (A4) and defining a new variable $z \equiv x \eta^{2} / r^{2}$, we have

$$
\begin{aligned}
\langle\bar{\psi} \psi\rangle_{s}^{d S}= & \frac{q}{\sqrt{2} \pi^{5 / 2} \alpha^{3}} \int_{0}^{\infty} d z z^{3 / 2} e^{z\left(1-r^{2} / \eta^{2}\right)} \\
& \times \operatorname{Im}\left[K_{1 / 2-i m \alpha}(z)\right] \mathcal{J}\left(q, a_{0}, z r^{2} / \eta^{2}\right),
\end{aligned}
$$

Following the procedure used in the Ref. [53] for the summation over $j$ in (3.11), we obtain

$$
\begin{aligned}
\mathcal{J}(q, & \left.a_{0}, w\right) \\
= & \frac{2}{q} e^{w}+\frac{4}{q} \sum_{k=1}^{p}(-1)^{k} \cos \left(2 k \pi a_{0}\right) \cos (k \pi / q) e^{w \cos (2 k \pi / q)} \\
& +\frac{4}{\pi} \int_{0}^{\infty} d x \frac{h\left(q, a_{0}, 2 x\right) \sinh x}{\cosh (2 q x)-\cos (q \pi)} e^{-w \cosh 2 x}
\end{aligned}
$$

where $p$ is an integer number defined by the relation $2 p<$ $q<2 p+2$ and

$$
\begin{aligned}
& h\left(q, a_{0}, 2 x\right) \\
& \quad=\sum_{\chi=+,-} \cos \left[q \pi\left(1 / 2+\chi a_{0}\right)\right] \sinh \left[2 q x\left(1 / 2-\chi a_{0}\right)\right] .
\end{aligned}
$$

In the case of $q<2$ the summation term in (3.14) is absent. Also for the special case of $q=2 p$, one should add the term 


$$
-(-1)^{q / 2} \frac{e^{-w}}{q} \sin \left(q \pi a_{0}\right)
$$

to the Eq. (3.14).

The first term in the right-hand side of the Eq. (3.14) provides the FC due only to the $\mathrm{dS}$ spacetime. The second and the third ones are the contributions due to the nontrivial topology of the cosmic string spacetime and the magnetic flux. Because here we are mostly interested in the contribution to the FC induced by the presence of the string, we shall discard the pure dS contribution, $\langle\bar{\psi} \psi\rangle_{d S}$, according to the expression below,

$$
\langle\bar{\psi} \psi\rangle_{s}=\langle\bar{\psi} \psi\rangle_{s}^{d S}-\langle\bar{\psi} \psi\rangle_{d S}
$$

It is important to note that the presence of cosmic string does not change the local geometry of dS spacetime for points away from the string. Consequently, the renormalization is needed only for the pure $\mathrm{dS}$ contribution. Therefore, the result is finite for $r \neq 0$ and is given by

$$
\begin{aligned}
\langle\bar{\psi} \psi\rangle_{s}= & \frac{2^{3 / 2}}{\pi^{5 / 2} \alpha^{3}}\left[\sum_{k=1}^{p}(-1)^{k} \cos \left(2 k \pi a_{0}\right) \cos (k \pi / q)\right. \\
& \times \int_{0}^{\infty} d z z^{3 / 2} e^{z\left[1-2\left(r^{2} / \eta^{2}\right) s_{k}^{2}\right]} \\
& +\frac{q}{\pi} \int_{0}^{\infty} d x \frac{h\left(q, a_{0}, 2 x\right) \sinh x}{\cosh (2 q x)-\cos (q \pi)} \\
& \left.\times \int_{0}^{\infty} d z z^{3 / 2} e^{z\left[1-2\left(r^{2} / \eta^{2}\right) c_{x}^{2}\right]}\right] \operatorname{Im}\left[K_{1 / 2-i m \alpha}(z)\right]
\end{aligned}
$$

where we have defined

$$
s_{k}=\sin (k \pi / q) \quad \text { and } \quad c_{x}=\cosh x
$$

Using the formula (A5) we can evaluate the integral over $z$ and also with the help of (A6) the final expression for the FC induced by the magnetic flux and the planar angle deficit reads

$$
\begin{aligned}
\langle\bar{\psi} \psi\rangle_{s}= & \frac{2^{3 / 2}}{\pi^{5 / 2} \alpha^{3}}\left\{\sum_{k=1}^{p}(-1)^{k} \cos \left(2 k \pi a_{0}\right) \cos (k \pi / q) \mathcal{G}_{0}\left(u_{0, k}\right)\right. \\
& \left.+\frac{q}{\pi} \int_{0}^{\infty} d x \frac{h\left(q, a_{0}, 2 x\right) \sinh x}{\cosh (2 q x)-\cos (q \pi)} \mathcal{G}_{0}\left(u_{0, x}\right)\right\} .
\end{aligned}
$$

In the above expression we have introduced the notation

$$
\mathcal{G}_{l}\left(u_{l, y}\right)=\operatorname{Im}\left[\mathcal{F}_{l}\left(u_{l, y}\right)\right]
$$

where
$\mathcal{F}_{l}\left(u_{l, y}\right)=\frac{\sqrt{\pi}}{2^{7 / 2}} \frac{\Gamma(3-i m \alpha) \Gamma(2+i m \alpha)}{u_{l, y}^{3-i m \alpha}} F\left(a, b ; c ; d_{l, y}\right)$,

with $F\left(a, b ; c ; d_{l, y}\right)$ being the hypergeometric function

$F\left(a, b ; c ; d_{l, y}\right) \equiv F\left(2-\frac{i m \alpha}{2}, \frac{3-i m \alpha}{2} ; 3 ; 1-u_{l, y}^{-2}\right)$

and

$u_{l, k}=\frac{l^{2} L^{2}}{2 \eta^{2}}+\frac{2 r^{2} s_{k}^{2}}{\eta^{2}}-1 \quad$ and $\quad u_{l, x}=\frac{l^{2} L^{2}}{2 \eta^{2}}+\frac{2 r^{2} c_{x}^{2}}{\eta^{2}}-1$

In the Eq. (3.20), we have (3.21) and (3.24) with $l=0$. One can notice that the string contribution in $\mathrm{FC}$ is an even function of the parameter $a_{0}$.

Now, let us consider some particular cases of the expression (3.20). For example, for a massless fermionic field, it is easy to see that the string part of the FC vanishes. This result is more evident looking at the expression (3.18).

Now, let us study the short and large distances from the string. For the region near the string, $r / \eta \ll 1$, it is more convenient to consider the expression (3.18). In this regime the dominant contribution to this expression comes from large values of $z$ and we can use the expansion of the Macdonald function for large arguments [49]. The leading term is given by

$$
\begin{aligned}
\langle\bar{\psi} \psi\rangle_{s} \approx & -\frac{m}{2 \pi^{2}}\left(\frac{\eta}{\alpha r}\right)^{2}\left\{\sum_{k=1}^{p}(-1)^{k} \frac{\cos \left(2 k \pi a_{0}\right) \cos (k \pi / q)}{s_{k}^{2}}\right. \\
& \left.+\frac{q}{\pi} \int_{0}^{\infty} d x \frac{c_{x}^{-2} h\left(q, a_{0}, 2 x\right) \tanh x}{\cosh (2 q x)-\cos (q \pi)}\right\} .
\end{aligned}
$$

It is important to have in mind that the ratio $r / \eta$ means the proper distance from the cosmic string in units of the dS curvature ratio $\alpha$. In the above expression there is a divergence with the second power of the proper distance matching exactly the behavior obtained in [44].

For large distances from the string, $r / \eta \gg 1$, the main contribution to the integral over $z$ in (3.18) comes from the lower limit of integration and we can use the asymptotic expression of the Macdonald function for small arguments. Considering the leading term, the string part of the FC behaves as

$$
\begin{aligned}
\langle\bar{\psi} \psi\rangle_{s} \approx & \frac{1}{\pi^{5 / 2} \alpha^{3}}\left(\frac{\eta}{r}\right)^{4} \operatorname{Im}\left\{\frac{\Gamma(2+i m \alpha) \Gamma(1 / 2-i m \alpha)}{2^{2 i m \alpha-1}}\left(\frac{\eta}{r}\right)^{i m \alpha}\right. \\
& \times\left[\sum_{k=1}^{p}(-1)^{k} \frac{\cos \left(2 k \pi a_{0}\right) \cos (k \pi / q)}{s_{k}^{4+2 i m \alpha}}\right. \\
& \left.\left.\times \frac{q}{\pi} \int_{0}^{\infty} d x \frac{c_{x}^{-4-2 i m \alpha} \sinh x h\left(q, a_{0}, 2 x\right)}{\cosh (2 q x)-\cos (q \pi)}\right]\right\}
\end{aligned}
$$



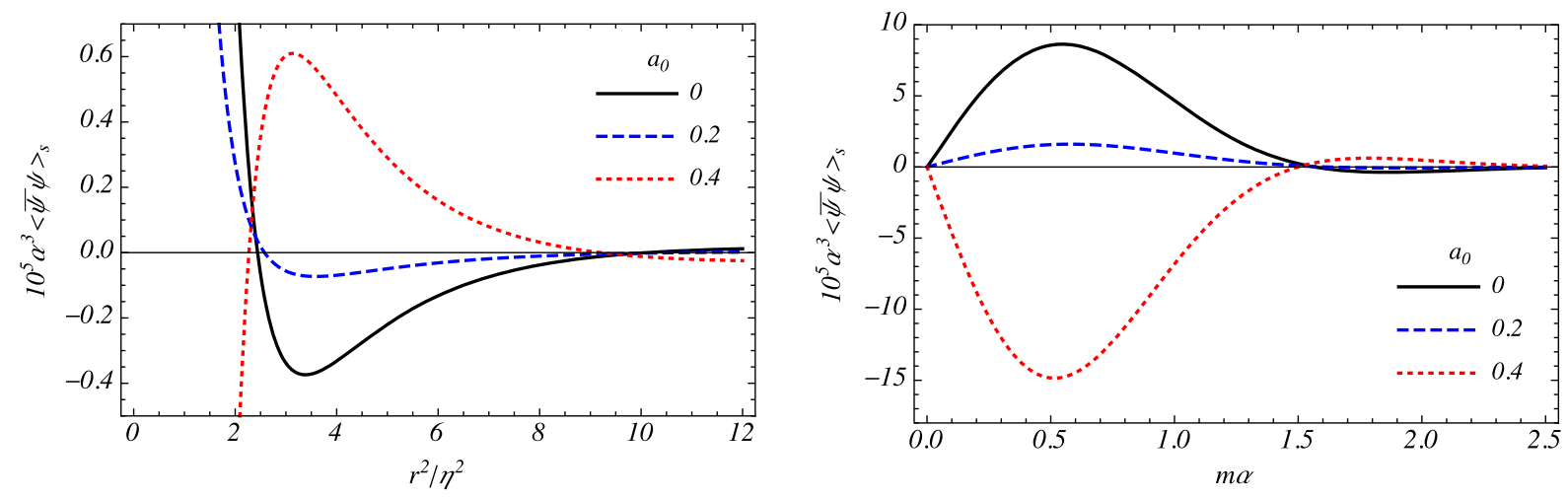

FIG. 1. Fermionic condensate induced by the magnetic flux and planar angle deficit as function of $r^{2} / \eta^{2}$ (left plot) for $m \alpha=2$ and the mass of the field (right plot) for $r^{2} \eta^{2}=2$. For both plots we adopted $q=1.5$.

The string part of the FC is exhibited as a function of the ratio $r^{2} / \eta^{2}$ in the left plot of Fig. 1, for different values of the parameter $a_{0}{ }^{1}$ In the right plot we show the same but now as a function of $m \alpha$, where we note that the FC vanishes in the limit of very massive fields. The results shown in this figure match the expected result for the case $a_{0}=0$, in the absence of the magnetic field [see Fig. 1 in [44]]. As one can see, the behavior of the string part of FC as a function of the proper distance and the mass changes dramatically depending on the value of $a_{0}$. With the parameters chosen for the figure, the threshold value is around $a_{0} \approx 0.22$.

Now, for the contribution of the FC induced by the compactification using the second term of the Abel-Plana summation formula we obtain

$$
\begin{aligned}
\langle\bar{\psi} \psi\rangle_{c}= & \frac{q \eta^{4}}{4 \pi^{3} \alpha^{3}}\left(\partial_{\eta}+\frac{1-2 i m \alpha}{\eta}\right) \int_{0}^{\infty} d p p \sum_{j}\left[J_{\beta_{1}}^{2}(p r)+J_{\beta_{2}}^{2}(p r)\right] \int_{p}^{\infty} d u K_{1 / 2-i m \alpha}\left(\eta \sqrt{u^{2}-p^{2}}\right) \\
& \times\left[K_{1 / 2-i m \alpha}\left(e^{i \pi} \eta \sqrt{u^{2}-p^{2}}\right)-K_{1 / 2-i m \alpha}\left(e^{-i \pi} \eta \sqrt{u^{2}-p^{2}}\right)\right] \sum_{\chi= \pm 1} \frac{1}{e^{L u+2 \pi i x \tilde{\beta}}-1},
\end{aligned}
$$

where we have used the relation

$$
\sqrt{( \pm i u)^{2}+p^{2}}= \begin{cases}\sqrt{p^{2}-u^{2}}, & \text { if } u<p \\ \pm i \sqrt{u^{2}-p^{2}}, & \text { if } u>p\end{cases}
$$

to show that the integral over $u$ in the interval $[0, p]$ vanishes while in the interval $[p, \infty]$ does not. Equations (A8) and (A9) help to write the part of the FC induced by the compactification as follows

$$
\begin{aligned}
\langle\bar{\psi} \psi\rangle_{c}= & -\frac{i q \eta^{4}}{2 \pi^{2} \alpha^{3}} \sum_{l=1}^{\infty} \cos (2 \pi l \tilde{\beta})\left(\partial_{\eta}+\frac{1-2 i m \alpha}{\eta}\right) \int_{0}^{\infty} d p p \sum_{j}\left[J_{\beta_{1}}^{2}(p r)+J_{\beta_{2}}^{2}(p r)\right] \\
& \times \int_{0}^{\infty} d \lambda \lambda \frac{e^{-l L \sqrt{\lambda^{2}+p^{2}}}}{\sqrt{\lambda^{2}+p^{2}}} K_{1 / 2-i m \alpha}(\eta \lambda)\left[I_{1 / 2-i m \alpha}(\eta \lambda)+I_{-1 / 2+i m \alpha}(\eta \lambda)\right],
\end{aligned}
$$

where $\lambda=\sqrt{u^{2}-p^{2}}$ and we have used the expansion $\left(e^{u}-1\right)^{-1}=\sum_{l=1}^{\infty} e^{-l u}$. For the further transformation of the above expression we apply the identity bellow,

\footnotetext{
${ }^{1}$ All the figures presented in this paper are obtained numerically using Mathematica.
}

$$
\frac{e^{-l L \sqrt{\lambda^{2}+p^{2}}}}{\sqrt{\lambda^{2}+p^{2}}}=\frac{2}{\sqrt{\pi}} \int_{0}^{\infty} d s e^{-\left(\lambda^{2}+p^{2}\right) s^{2}-l^{2} L^{2} /\left(4 s^{2}\right)} .
$$

Substituting the above integral representation into the Eq. (3.29), we are able to evaluate the integration over $p$ and $\lambda$. Therefore, for the part induced by the compactification we obtain 


$$
\begin{aligned}
\langle\bar{\psi} \psi\rangle_{c}= & \frac{\sqrt{2} q}{\pi^{5 / 2} \alpha^{3}} \sum_{l=1}^{\infty} \cos (2 \pi l \tilde{\beta}) \int_{0}^{\infty} d z z^{3 / 2} e^{z\left[1-r^{2} / \eta^{2}-l^{2} L^{2} /\left(2 \eta^{2}\right)\right]} \\
& \times \operatorname{Im}\left[K_{1 / 2-i m \alpha}(z)\right] \mathcal{J}\left(q, a_{0}, z r^{2} / \eta^{2}\right)
\end{aligned}
$$

where the function $\mathcal{J}\left(q, a_{0}, z r^{2} / \eta^{2}\right)$ is given by the Eq. (3.14). As a result, the final expression for the FC induced by the compactification is given by

$$
\begin{aligned}
\langle\bar{\psi} \psi\rangle_{c}= & \frac{2^{5 / 2}}{\pi^{5 / 2} \alpha^{3}} \sum_{l=1}^{\infty} \cos (2 \pi l \tilde{\beta}) \\
& \times\left\{\sum_{k=0}^{p}(-1)^{k} \cos \left(2 k \pi a_{0}\right) \cos (k \pi / q) \mathcal{G}_{l}\left(u_{l, k}\right)\right. \\
& \left.+\frac{q}{\pi} \int_{0}^{\infty} d x \frac{h\left(q, a_{0}, 2 x\right) \sinh x}{\cosh (2 q x)-\cos (q \pi)} \mathcal{G}_{l}\left(u_{l, x}\right)\right\},
\end{aligned}
$$

with the notation (3.21) and (3.24). It is easy to see that the compactification part as well as the string part of FC is zero for the massless fermion.

The compactification part of the FC can be decomposed as

$$
\langle\bar{\psi} \psi\rangle_{c}=\langle\bar{\psi} \psi\rangle_{c}^{(0)}+\langle\bar{\psi} \psi\rangle_{c}^{\left(q, a_{0}\right)}
$$

The first term on the right-hand side of the above decomposition is the $k=0$ term of (3.32) with the coefficient $1 / 2$. This is a pure topological term dependent only on the compactification and the curvature of the dS spacetime and independent of the radial coordinate and the magnetic flux. This contribution is given by

$$
\langle\bar{\psi} \psi\rangle_{c}^{(0)}=\frac{2^{3 / 2}}{\pi^{5 / 2} \alpha^{3}} \sum_{l=1}^{\infty} \cos (2 \pi i \tilde{\beta}) \mathcal{G}_{l}\left(u_{l, 0}\right) .
$$

The second term on the right-hand side of (3.33) is given by

$$
\begin{aligned}
\langle\bar{\psi} \psi\rangle_{c}^{\left(q, a_{0}\right)}= & \frac{2^{5 / 2}}{\pi^{5 / 2} \alpha^{3}} \sum_{l=1}^{\infty} \cos (2 \pi l \tilde{\beta}) \\
& \times\left\{\sum_{k=1}^{p}(-1)^{k} \cos \left(2 k \pi a_{0}\right) \cos (k \pi / q) \mathcal{G}_{l}\left(u_{l, k}\right)\right. \\
& \left.+\frac{q}{\pi} \int_{0}^{\infty} d x \frac{h\left(q, a_{0}, 2 x\right) \sinh x}{\cosh (2 q x)-\cos (q \pi)} \mathcal{G}_{l}\left(u_{l, x}\right)\right\},
\end{aligned}
$$

which is the contribution to the FC induced by the compactification and the magnetic flux.

We now evaluate some asymptotic expressions of the previous equation. Considering $r / \eta \ll 1$, near the string, the leading order reads

$$
\begin{aligned}
\langle\bar{\psi} \psi\rangle_{c}^{\left(q, a_{0}\right)} \approx & \frac{q \cos \left[q \pi\left(1 / 2+\left|a_{0}\right|\right)\right] \Gamma\left(1 / 2-q\left(1 / 2-\left|a_{0}\right|\right)\right)}{\pi^{7 / 2} \alpha^{3}}\left(\frac{r}{\sqrt{2} \eta}\right)^{q\left(1-2\left|a_{0}\right|\right)-1} \\
& \times \sum_{l=1}^{\infty} \cos (2 \pi l \tilde{\beta}) \operatorname{Im}\left\{\frac{\Gamma(2+i m \alpha) \Gamma\left(3 / 2-i m \alpha+q\left(1-2\left|a_{0}\right|\right)\right)}{2^{1+i m \alpha} \Gamma(2-i m \alpha)\left(l L /(\sqrt{2} \eta)^{3-i m \alpha+q\left(1-2\left|a_{0}\right|\right)}\right)}\right\} .
\end{aligned}
$$

The FC is finite on the string for $\left|a_{0}\right|=(1-1 / q) / 2$, vanishes for $\left|a_{0}\right|<(1-1 / q) / 2$ and diverges for $\left|a_{0}\right|>(1-1 / q) / 2$. Note that in the absence of axial magnetic flux, the FC vanishes on the string's core.

For large values of $L / \eta$, we use (A7), and to the leading order we find

$$
\begin{aligned}
\langle\bar{\psi} \psi\rangle_{c}^{\left(q, a_{0}\right)} \approx & \frac{2^{5 / 2}}{\pi^{5 / 2} \alpha^{3}}\left(\frac{\eta}{L}\right)^{4} \sum_{l=1}^{\infty} \cos (2 \pi i \tilde{\beta}) \operatorname{Im}\left\{\frac{\Gamma(1 / 2-i m \alpha) \Gamma(2+i m \alpha)}{2^{i m \alpha+1 / 2}}\left(\frac{\eta}{L}\right)^{2 i m \alpha}\right. \\
& \left.\times\left[\sum_{k=1}^{p} \frac{(-1)^{k} \cos \left(2 k \pi a_{0}\right) \cos (k \pi / q)}{\left(\frac{l^{2}}{2}+\frac{r^{2} s_{k}^{2}}{L^{2}}\right)^{2+i m \alpha}}+\frac{q}{\pi} \int_{0}^{\infty} d x \frac{h\left(q, a_{0}, 2 x\right) \sinh x}{\cosh (2 q x)-\cos (q \pi)}\left(\frac{l^{2}}{2}+\frac{r^{2} c_{x}^{2}}{L^{2}}\right)^{-2-i m \alpha}\right]\right\} .
\end{aligned}
$$

As can be seen the FC, considering the length of the compact dimension much larger than the curvature of the dS spacetime, presents a fourth power decay.

Figure 2 shows the behavior of the FC induced by the compactification as a function of $L / \eta$ (left plot) and $\tilde{\beta}$ (right plot). ${ }^{2}$ As one expects, in the limit $L \rightarrow \infty$, the compactification contribution of FC vanishes.

\footnotetext{
${ }^{2}$ In the summation over $l$, due to the rapid convergence of the series in our numerical calculation, we consider only the dominant contributions given by small $l$.
}

\section{ENERGY-MOMENTUM TENSOR}

Another important physical quantity which characterizes the quantum vacuum is the VEV of the energy-momentum tensor. Considering a charged fermionic field this VEV can be evaluated considering the summation formula

$$
\begin{aligned}
\left\langle 0\left|T_{\mu \nu}\right| 0\right\rangle= & \frac{i}{2} \sum_{\sigma}\left[\bar{\psi}_{\sigma}^{(-)}(x) \gamma_{(\mu} \mathcal{D}_{\nu)} \psi_{\sigma}^{(-)}(x)\right. \\
& \left.-\left(\mathcal{D}_{(\mu} \bar{\psi}_{\sigma}^{(-)}(x)\right) \gamma_{\nu)} \psi_{\sigma}^{(-)}(x)\right]
\end{aligned}
$$



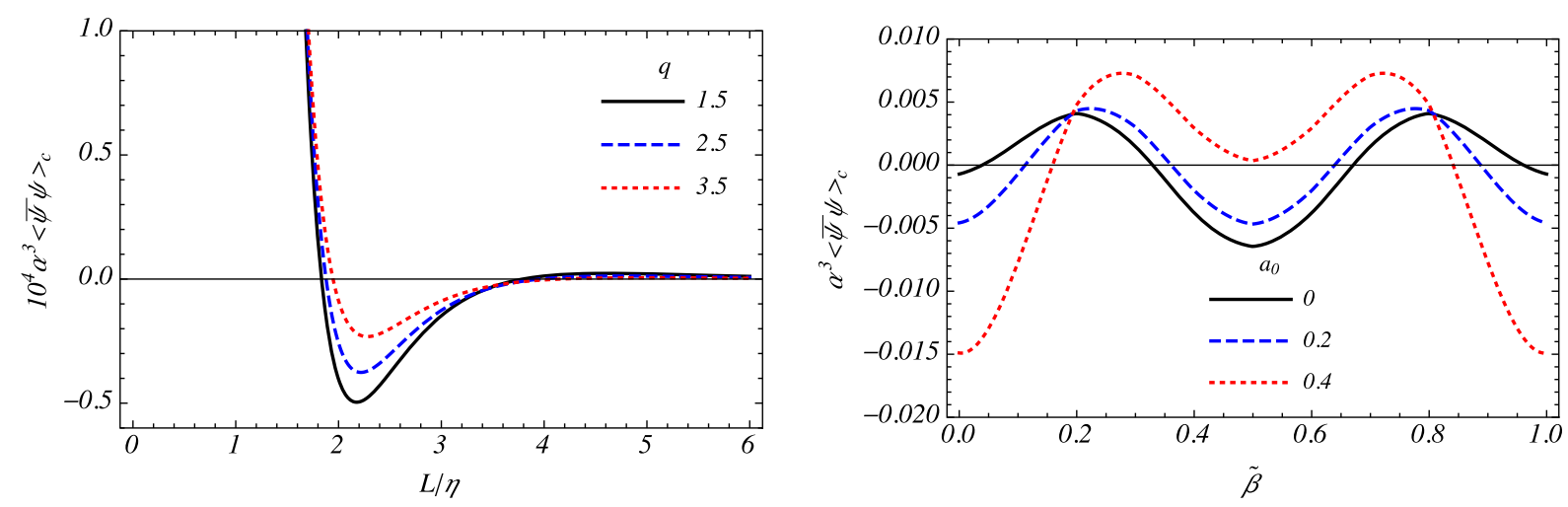

FIG. 2. FC induced by the magnetic flux, the planar angle deficit and the compactification. In the left plot the behavior of the FC is shown as a function of $L / \eta$ considering three different values of $q, a_{0}=0.25, r / \eta=0.75$ and $\tilde{\beta}=0.25$. In the right plot we have the same quantity, but as a function of $\tilde{\beta}$ considering three different values of $a_{0}$ for $q=1.5, r / \eta=0.5$ and $L / \eta=0.75$. In both graphs $m \alpha=2$.

where we consider the negative energy eigenspinors given by the Eq. (2.6) and $\mathcal{D}_{\mu} \bar{\psi}=\partial_{\mu} \bar{\psi}-i e A_{\mu} \bar{\psi}-\bar{\psi} \Gamma_{\mu}$. In the above expression, the brackets in the index expression mean the symmetrization over the enclosed indexes.

The geometry that we have considered allows us to decompose the VEV of the energy-momentum tensor in the following way

$$
\left\langle 0\left|T_{\mu}^{\nu}\right| 0\right\rangle=\left\langle 0\left|T_{\mu}^{\nu}\right| 0\right\rangle_{s}^{d S}+\left\langle 0\left|T_{\mu}^{\nu}\right| 0\right\rangle_{c},
$$

where the first term is the contribution induced by the cosmic string and the curvature of the de Sitter spacetime, while the second one is the contribution induced by the compactification.

\section{A. Energy density}

Let us start with the evaluation of the vacuum energy density, $\left\langle 0\left|T_{0}^{0}\right| 0\right\rangle$. Using the expression for the eigenfunctions given by the Eq. (2.6), and taking into account that $\Gamma_{0}=0$ [44], after long but straightforward calculations, we obtain

$$
\begin{aligned}
\left\langle 0\left|T_{0}^{0}\right| 0\right\rangle= & -\frac{q \eta^{5}}{4 \pi^{2} \alpha^{4} L} \sum_{l=-\infty}^{\infty} \sum_{j} \int_{0}^{\infty} d p p\left[J_{\beta_{1}}^{2}(p r)+J_{\beta_{2}}^{2}(p r)\right] \\
& \times\left[4 \gamma^{2}+\hat{S}_{\eta}\right] K_{1 / 2-i m \alpha}(i \gamma \eta) K_{1 / 2-i m \alpha}(-i \gamma \eta),
\end{aligned}
$$

where we have defined the operator

$$
\hat{S}_{\eta}=\partial_{\eta}^{2}+\frac{2}{\eta} \partial_{\eta}+\frac{4 i m \alpha(1 / 2-i m \alpha)}{\eta^{2}} .
$$

The expression for the energy density can be decomposed as indicated by (4.2). The Abel-Plana summation formula (3.6) allows us to solve the summation over the quantum number $l$ in the expression (4.3). For the first term inside the square brackets we take

$$
f(u)=K_{1 / 2-i m \alpha}(i \gamma \eta) K_{1 / 2-i m \alpha}(-i \gamma \eta),
$$

and for the second one we take

$$
f(u)=\gamma^{2} K_{1 / 2-i m \alpha}(i \gamma \eta) K_{1 / 2-i m \alpha}(-i \gamma \eta),
$$

considering $g(u)=1$ for both terms. The contribution induced by the cosmic string and the curvature of the dS spacetime, taking the first term on the right-hand side of (3.6), is given by

$$
\begin{aligned}
\left\langle 0\left|T_{0}^{0}\right| 0\right\rangle_{s}^{d S}= & -\frac{q \eta^{5}}{4 \pi^{3} \alpha^{4}} \sum_{j} \int_{0}^{\infty} d p p\left[J_{\beta_{1}}^{2}(p r)+J_{\beta_{2}}^{2}(p r)\right] \\
& \times\left\{\hat{S}_{\eta} \int_{0}^{\infty} d k K_{1 / 2-i m \alpha}(i \gamma \eta) K_{1 / 2-i m \alpha}(-i \gamma \eta)\right. \\
& \left.+4 \int_{0}^{\infty} d k \gamma^{2} K_{1 / 2-i m \alpha}(i \gamma \eta) K_{1 / 2-i m \alpha}(-i \gamma \eta)\right\} .
\end{aligned}
$$

Thanks to the representation (A3) the integration over the variable $k$ can be performed following a similar procedure adopted in the Appendix A of the Ref. [44]. As a result we have

$$
\begin{aligned}
\left\langle 0\left|T_{0}^{0}\right| 0\right\rangle_{s}^{d S}= & -\frac{q(\eta / r)^{5}}{2^{3 / 2} \pi^{5 / 2} \alpha^{4}} \int_{0}^{\infty} d x x^{3 / 2} e^{-x} \mathcal{J}\left(q, a_{0}, x\right) \\
& \times\left.\hat{F}_{u} e^{u} K_{1 / 2-i m \alpha}(u)\right|_{u=x \eta^{2} / r^{2}}
\end{aligned}
$$

where we have defined the operator

$\hat{F}_{u}=u \partial_{u}^{2}+(3 / 2-2 u) \partial_{u}-2+\frac{i m \alpha(1 / 2-i m \alpha)}{u}$.

From the properties of the Macdonald functions, one can see that 


$$
\hat{F}_{u} e^{u} K_{1 / 2-i m \alpha}(u)=-\frac{1}{2} e^{u}\left[K_{1 / 2-i m \alpha}(u)+\left[K_{-1 / 2+i m \alpha}(u)\right] .\right.
$$

Taking into account the previous results we find

$$
\begin{aligned}
\left\langle 0\left|T_{0}^{0}\right| 0\right\rangle_{s}^{d S}= & \frac{q \alpha^{-4}}{2^{3 / 2} \pi^{5 / 2}} \int_{0}^{\infty} d z z^{3 / 2} e^{z\left(1-r^{2} / \eta^{2}\right)}\left[\operatorname{Re} K_{1 / 2-i m \alpha}(z)\right] \\
& \times \mathcal{J}\left(q, a_{0}, z r^{2} / \eta^{2}\right)
\end{aligned}
$$

where $\mathcal{J}\left(q, a_{0}, z r^{2} / \eta^{2}\right)$ is given by (3.14). Following a similar procedure as in (3.17) to extract the dS part for the FC and using (A5) and (A6), we find the final expression for the VEV of the energy density induced by the cosmic string in $\mathrm{dS}$ space

$$
\begin{aligned}
& \left\langle 0\left|T_{0}^{0}\right| 0\right\rangle_{s} \\
& =\frac{1}{\pi^{2} \alpha^{4}} \sqrt{\frac{2}{\pi}}\left[\sum_{k=1}^{p}(-1)^{k} \cos \left(2 k \pi a_{0}\right) \cos (k \pi / q) \mathcal{M}_{0}\left(u_{0, k}\right)\right. \\
& \left.\quad+\frac{q}{\pi} \int_{0}^{\infty} d x \frac{\sinh (x) h\left(q, a_{0}, 2 x\right)}{\cosh (2 q x)-\cos (q \pi)} \mathcal{M}_{0}\left(u_{0, x}\right)\right]
\end{aligned}
$$

with the notation

$$
\mathcal{M}_{l}\left(u_{l, y}\right)=\operatorname{Re}\left[\mathcal{F}_{l}\left(u_{l, y}\right)\right]
$$

where we have defined $\mathcal{F}_{l}$ and $u_{l, y}$ in (3.21)-(3.24). We note that the energy density induced by the cosmic string is an even function of $a_{0}$ and depends on the ratio $r / \eta$.

Now, we focus on the compactification contribution to the VEV of the energy density. From the second term in the Abel Plana summation formula (3.6) and considering again (3.28), we get

$$
\begin{aligned}
\left\langle 0\left|T_{0}^{0}\right| 0\right\rangle_{c}= & -\frac{i q \eta^{5}}{8 \pi^{3} \alpha^{4}} \sum_{j} \int_{0}^{\infty} d p p\left[J_{\beta_{1}}^{2}(p r)+J_{\beta_{2}}^{2}(p r)\right] \int_{p}^{\infty} d u K_{1 / 2-i m \alpha}(\eta \lambda) \\
& \times\left[\hat{S}_{\eta}-4 \lambda^{2}\right]\left[K_{1 / 2-i m \alpha}\left(e^{i \pi} \eta \lambda\right)-K_{1 / 2-i m \alpha}\left(e^{-i \pi} \eta \lambda\right)\right] \sum_{\chi= \pm 1}\left(e^{L u+2 \pi i \chi \tilde{\beta}}-1\right)^{-1} .
\end{aligned}
$$

In the above integral we considered $\lambda=\sqrt{u^{2}-p^{2}}$, as before. By using again the Eqs. (A8) and (A9) besides the expansion $\left(e^{u}-1\right)^{-1}=\sum_{l=1}^{\infty} e^{-l u}$, we obtain

$$
\begin{aligned}
\left\langle 0\left|T_{0}^{0}\right| 0\right\rangle_{c}= & -\frac{i q \eta^{5}}{4 \pi^{2} \alpha^{4}} \sum_{l=1}^{\infty} \cos (2 \pi l \tilde{\beta}) \sum_{j} \int_{0}^{\infty} d p p\left[J_{\beta_{1}}^{2}(p r)+J_{\beta_{2}}^{2}(p r)\right] \int_{0}^{\infty} \frac{d \lambda}{\sqrt{\lambda^{2}+p^{2}}} \\
& \times\left[\lambda \hat{S}(\eta, \alpha)-4 \lambda^{3}\right] K_{1 / 2-i m \alpha}(\eta \lambda)\left[I_{1 / 2-i m \alpha}(\eta \lambda)+I_{-1 / 2+i m \alpha}(\eta \lambda)\right] e^{-l L \sqrt{\lambda^{2}+p^{2}}} .
\end{aligned}
$$

One can use the identity (3.30) which allows to solve the integration over $p$ as well as the integration over $\lambda$ knowing $\lambda^{3} e^{-\lambda^{2} s^{2}}=-\lambda \partial_{s^{2}} e^{-\lambda^{2} s^{2}}$. Doing so and considering similar transformations that we have done in the evaluation of the contribution induced by the compactification for the FC, we find

$$
\left\langle 0\left|T_{0}^{0}\right| 0\right\rangle_{c}=-\left.\frac{q \eta^{5}}{4 \pi^{5 / 2} \alpha^{4}} \sum_{l=1}^{\infty} \cos (2 \pi l \tilde{\beta}) \int_{0}^{\infty} d s s^{-6} e^{-\frac{l^{2} L^{2}}{4 s^{2}}-\frac{r^{2}}{2 s^{2}}} \mathcal{J}\left(q, a_{0}, r^{2} /\left(2 s^{2}\right)\right) \hat{F}_{u} e^{u} K_{1 / 2-i m \alpha(u)}\right|_{u=\eta^{2} / 2 s^{2}}
$$

where the operator $\hat{F}_{u}$ was defined in the Eq. (4.9). The next step, we consider (4.10) and (3.14) to obtain the final expression for the energy density induced by the compactification which is given by

$$
\begin{aligned}
\left\langle 0\left|T_{0}^{0}\right| 0\right\rangle_{c}= & \frac{2^{3 / 2}}{\pi^{5 / 2} \alpha^{4}} \sum_{l=1}^{\infty} \cos (2 \pi l \tilde{\beta})\left[\sum_{k=0}^{p}(-1)^{k} \cos \left(2 k \pi a_{0}\right) \cos (k \pi / q) \mathcal{M}_{l}\left(u_{l, k}\right)\right. \\
& \left.+\frac{q}{\pi} \int_{0}^{\infty} d x \frac{\sinh (x) h\left(q, a_{0}, 2 x\right)}{\cosh (2 q x)-\cos (q \pi)} \mathcal{M}_{l}\left(u_{l, x}\right)\right]
\end{aligned}
$$

where the prime on the summation means that the $k=0$ term has the coefficient $1 / 2$. 


\section{B. Radial stress}

Now, let us consider the VEV of the radial part of the stress-energy tensor. Taking into account $\Gamma_{r}$ in the Eq. (4.1) as well as considering the modes (2.6), we have

$$
\begin{aligned}
\left\langle 0\left|T_{1}^{1}\right| 0\right\rangle= & \frac{q \eta^{5}}{\pi^{2} \alpha^{4} L} \sum_{l=-\infty}^{\infty} \int_{0}^{\infty} d p p^{3} \sum_{j} \epsilon_{j}\left[J_{\beta_{1}}(p r) J_{\beta_{2}}^{\prime}(p r)-J_{\beta_{1}}^{\prime}(p r) J_{\beta_{2}}(p r)\right] \\
& \times \operatorname{Re}\left[K_{1 / 2-i m \alpha}(i \gamma \eta) K_{1 / 2-i m \alpha}(-i \gamma \eta)\right],
\end{aligned}
$$

where the prime in the Bessel functions means derivative with respect to the argument. Using the recurrent relation (A11) for the Bessel functions we obtain

$$
\begin{aligned}
\left\langle 0\left|T_{1}^{1}\right| 0\right\rangle= & \frac{q \eta^{5}}{\pi^{2} \alpha^{4} L} \sum_{l=-\infty}^{\infty} \int_{0}^{\infty} d p p^{3} \sum_{j}\left[J_{\beta_{1}}^{2}(p r)+J_{\beta_{2}}^{2}(p r)-\frac{2 \beta_{1}+\epsilon_{j}}{p r} J_{\beta_{1}}(p r) J_{\beta_{2}}(p r)\right] \\
& \times \operatorname{Re}\left[K_{1 / 2-i m \alpha}(i \gamma \eta) K_{1 / 2-i m \alpha}(-i \gamma \eta)\right] .
\end{aligned}
$$

We shall use the Abel-Plana summation formula to solve the summation over $l$ which allows us to decompose the radial stress as (4.2). We can use the (3.6) with $g(u)=1$ and $f(u)$ being the real part of (3.8). From the first term of the right-hand side of the Abel-Plana formula (3.6), the contribution coming from the curvature of the dS spacetime and the cosmic string can be written as

$$
\begin{aligned}
\left\langle 0\left|T_{1}^{1}\right| 0\right\rangle_{s}^{d S}= & \frac{q \eta^{5}}{\pi^{3} \alpha^{4}} \sum_{j} \int_{0}^{\infty} d p p^{3} \int_{0}^{\infty} d k \operatorname{Re}\left[K_{1 / 2-i m \alpha}(i \gamma \eta) K_{1 / 2-i m \alpha}(-i \gamma \eta)\right] \\
& \times\left[J_{\beta_{1}}^{2}(p r)+J_{\beta_{2}}^{2}(p r)-\frac{2 \beta_{1}+\epsilon_{j}}{p r} J_{\beta_{1}}(p r) J_{\beta_{2}}(p r)\right] .
\end{aligned}
$$

Combining the relations (A3), (A12), and (A13) where $x=r^{2} /\left(4 u \eta^{2} \sinh ^{2} y\right)$, the final expression for the radial stress induced by the string and the dS curvature is given by

$$
\left\langle 0\left|T_{1}^{1}\right| 0\right\rangle_{s}^{d S}=\frac{q \alpha^{-4}}{2^{3 / 2} \pi^{5 / 2}} \int_{0}^{\infty} d z z^{3 / 2} e^{z\left(1-r^{2} / \eta^{2}\right)} \operatorname{Re}\left[K_{1 / 2-i m \alpha}(z)\right] \mathcal{J}\left(q, a_{0}, z r^{2} / \eta^{2}\right)
$$

From this expression we can note that

$$
\left\langle 0\left|T_{1}^{1}\right| 0\right\rangle_{s}^{d S}=\left\langle 0\left|T_{0}^{0}\right| 0\right\rangle_{s}^{d S}
$$

Now, for the radial stress induced by the compactification, considering the second term on the right-hand side of the Abel-Plana summation formula (3.6) we have

$$
\begin{aligned}
\left\langle 0\left|T_{1}^{1}\right| 0\right\rangle_{c}= & \frac{q \eta^{5}}{\pi^{2} \alpha^{4}} \sum_{l=1}^{\infty} \cos (2 \pi l \tilde{\beta}) \sum_{j} \int_{0}^{\infty} d p p^{3}\left[J_{\beta_{1}}^{2}(p r)+J_{\beta_{2}}^{2}(p r)-\frac{2 \beta_{1}+\epsilon_{j}}{p r} J_{\beta_{1}}(p r) J_{\beta_{2}}(p r)\right] \\
& \times \int_{0}^{\infty} \frac{d \lambda \lambda}{\sqrt{\lambda^{2}+p^{2}}} \operatorname{Re}\left\{K_{1 / 2-i m \alpha}(\eta \lambda)\left[I_{1 / 2-i m \alpha}(\eta \lambda)+I_{-1 / 2+i m \alpha}(\eta \lambda)\right]\right\} e^{-l L \sqrt{\lambda^{2}+p^{2}}}
\end{aligned}
$$

where we have used the expansion $\left(e^{u}-1\right)^{-1}=\sum_{l=1}^{\infty} e^{-l u}$ besides the Eqs. (A8) and (A9). Considering the integral representation (3.30), the integral over $\lambda$ can be evaluated directly and the result is

$$
\begin{aligned}
\left\langle 0\left|T_{1}^{1}\right| 0\right\rangle_{c}= & \frac{q \eta^{5}}{\pi^{5 / 2} \alpha^{4}} \sum_{l=1}^{\infty} \cos (2 \pi i \tilde{\beta}) \sum_{j} \int_{0}^{\infty} d s \frac{e^{-l^{2} L^{2} /\left(4 s^{2}\right)+\eta^{2} /\left(2 s^{2}\right)}}{s^{2}} \operatorname{Re}\left[K_{1 / 2-i m \alpha}\left(\eta^{2} / 2 s^{2}\right)\right] \\
& \times \int_{0}^{\infty} d p p^{3} e^{-p^{2} s^{2}}\left[J_{\beta_{1}}^{2}(p r)+J_{\beta_{2}}^{2}(p r)-\frac{2 \beta_{1}+\epsilon_{j}}{p r} J_{\beta_{1}}(p r) J_{\beta_{2}}(p r)\right]
\end{aligned}
$$


To solve the integral over $p$ we make similar considerations done for the contribution induced by the curvature of the dS spacetime and the cosmic string which results in

$$
\begin{aligned}
\left\langle 0\left|T_{1}^{1}\right| 0\right\rangle_{c}= & \frac{q \alpha^{-4}}{2^{1 / 2} \pi^{5 / 2}} \sum_{l=1}^{\infty} \cos (2 \pi l \tilde{\beta}) \\
& \times \int_{0}^{\infty} d z z^{3 / 2} e^{z\left(1-r^{2} / \eta^{2}-l^{2} L^{2} /\left(2 \eta^{2}\right)\right)} \operatorname{Re}\left[K_{1 / 2-i m \alpha}(z)\right] \\
& \times \mathcal{J}\left(q, a_{0}, z r^{2} / \eta^{2}\right)
\end{aligned}
$$

As in the case of the string contribution, we note that

$$
\left\langle 0\left|T_{1}^{1}\right| 0\right\rangle_{c}=\left\langle 0\left|T_{0}^{0}\right| 0\right\rangle_{c} .
$$

\section{Azimuthal stress}

Our next step is the evaluation of the azimuthal stress. In order to proceed we take into account that [44]

$$
\Gamma_{2}=-\frac{1}{2 \alpha} \gamma^{0} \gamma_{2}+\frac{1-q}{2} \gamma^{(1)} \gamma^{(2)} .
$$

Using the negative energy eingenfunction in the mode sum (4.1), one finds

$$
\begin{aligned}
& \left\langle 0\left|T_{2}^{2}\right| 0\right\rangle \\
& =\frac{2 q^{2} \eta^{5}}{\pi^{2} \alpha^{4} L r^{2}} \sum_{l=-\infty}^{\infty} \sum_{j}(j+a)\left(\epsilon_{j} \beta_{1}-\frac{r}{2} \partial_{r}\right) \int_{0}^{\infty} d p p J_{\beta_{1}}^{2}(p r) \\
& \quad \times \operatorname{Re}\left[K_{1 / 2-i m \alpha}(i \gamma \eta) K_{1 / 2-i m \alpha}(-i \gamma \eta)\right]
\end{aligned}
$$

where we have used the relation (A14).

Again, using the Abel-Plana summation formula, considering $g(u)=1$ and $f(u)$ as the real part of (3.8), we are able to decompose this component of the energy-momentum tensor into two contributions. For the contribution induced by the curvature of the dS spacetime and the cosmic string, and considering similar transformations previously done, we have

$$
\begin{aligned}
& \left\langle 0\left|T_{2}^{2}\right| 0\right\rangle_{s}^{d S} \\
& =\frac{q^{2}}{2^{1 / 2} \pi^{5 / 2} \alpha^{4}} \sum_{j}(j+a) \int_{0}^{\infty} d z z^{3 / 2} e^{z} \operatorname{Re}\left[K_{1 / 2-i m \alpha}(z)\right] \\
& \quad \times\left.\left(\frac{\epsilon_{j} \beta_{1}}{x}-\partial_{x}\right) e^{-x} I_{\beta_{1}}(x)\right|_{x=z r^{2} / \eta^{2}}
\end{aligned}
$$

Following the representations (A15) and (A13), one obtains

$$
\begin{aligned}
\langle 0| & T_{2}^{2}|0\rangle_{s}^{d S} \\
= & \frac{q}{2^{1 / 2} \pi^{5 / 2} \alpha^{4}} \int_{0}^{\infty} d z z^{3 / 2} e^{z\left(1-r^{2} / \eta^{2}\right)} \operatorname{Re}\left[K_{1 / 2-i m \alpha}(z)\right] \\
& \times\left(z \partial_{z}-z r^{2} / \eta^{2}+1 / 2\right) \sum_{j}\left[I_{\beta_{1}}\left(z r^{2} / \eta^{2}\right)+I_{\beta_{2}}\left(z r^{2} / \eta^{2}\right)\right],
\end{aligned}
$$

knowing $q(j+a)=\epsilon_{j} \beta_{1}+1 / 2$. Writing the parameter $a$ as indicated by (2.9) and comparing the above equation with the Eq. (4.21), we note that following relation holds

$$
\left\langle 0\left|T_{2}^{2}\right| 0\right\rangle_{s}^{d S}=\partial_{r}\left[r\left\langle 0\left|T_{1}^{1}\right| 0\right\rangle_{s}^{d S}\right] .
$$

Using the corresponding radial stress, and extracting the divergent part, we find the azimuthal one in the following form

$$
\begin{aligned}
\left\langle 0\left|T_{2}^{2}\right| 0\right\rangle_{s}= & \frac{1}{2^{1 / 2} \pi^{5 / 2} \alpha^{4}}\left[\sum_{k=1}^{p}(-1)^{k} \cos \left(2 k \pi a_{0}\right) \cos (k \pi / q) \mathcal{M}_{0}^{(\phi)}\left(u_{0, k}, \frac{r}{\eta} s_{k}\right)\right. \\
& \left.+\frac{q}{\pi} \int_{0}^{\infty} d x \frac{\sinh (x) h\left(q, a_{0}, 2 x\right)}{\cosh (2 q x)-\cos (q \pi)} \mathcal{M}_{0}^{(\phi)}\left(u_{0, x}, \frac{r}{\eta} c_{x}\right)\right],
\end{aligned}
$$

where we define the notation

$$
\begin{aligned}
\mathcal{M}_{l}^{(\phi)}\left(u_{l, y}, v\right)= & \frac{\sqrt{\pi}}{2^{5 / 2}} \operatorname{Re}\left\{\frac { \Gamma ( 3 - i m \alpha ) \Gamma ( 2 + i m \alpha ) } { u _ { l , y } ^ { 6 - i m \alpha } } \left[u_{l, y}^{2}\left(\frac{l^{2} L^{2}}{2 \eta^{2}}+2(2 i m \alpha-5) v^{2}-1\right) F\left(a, b ; c ; d_{l, y}\right)\right.\right. \\
& \left.\left.+\frac{2}{3}(3-i m \alpha)(4-i m \alpha) v^{2} F\left(a+1, b+1 ; c+1 ; d_{l, y}\right)\right]\right\} .
\end{aligned}
$$

taking $l=0$.

Considering the second term in the Abel-Plana summation formula and doing similar transformations, for the contribution of the azimuthal stress induced by the compactification we find 


$$
\begin{aligned}
\left\langle 0\left|T_{2}^{2}\right| 0\right\rangle_{c}= & \frac{2^{1 / 2} q}{\pi^{5 / 2} \alpha^{4}} \sum_{l=1} \cos (2 \pi \tilde{\beta}) \int_{0}^{\infty} d z z^{3 / 2} e^{-z\left(l^{2} L^{2} /\left(2 \eta^{2}\right)+r^{2} / \eta^{2}\right)} \operatorname{Re}\left[K_{1 / 2-i m \alpha}(z)\right] \\
& \times\left(z \partial_{z}-z r^{2} / \eta^{2}+1 / 2\right) \sum_{j}\left[I_{\beta_{1}}\left(z r^{2} / \eta^{2}\right)+I_{\beta_{2}}\left(z r^{2} / \eta^{2}\right)\right] .
\end{aligned}
$$

Again, it is easy to show that

$$
\left\langle 0\left|T_{2}^{2}\right| 0\right\rangle_{c}=\partial_{r}\left[r\left\langle 0\left|T_{1}^{1}\right| 0\right\rangle_{c}\right]
$$

which leads to the final expression for the azimuthal stress induced by the compactification as

$$
\begin{aligned}
\left\langle 0\left|T_{2}^{2}\right| 0\right\rangle_{c}= & \frac{2^{1 / 2}}{\pi^{5 / 2} \alpha^{4}} \sum_{l=1}^{\infty} \cos (2 \pi l \tilde{\beta})\left[\sum_{k=0}^{p}(-1)^{k} \cos \left(2 k \pi a_{0}\right) \cos (k \pi / q) \mathcal{M}_{l}^{(\phi)}\left(u_{l, k}, \frac{r}{\eta} s_{k}\right)\right. \\
& \left.+\frac{q}{\pi} \int_{0}^{\infty} d x \frac{\sinh (x) h\left(q, a_{0}, 2 x\right)}{\cosh (2 q x)-\cos (q \pi)} \mathcal{M}_{l}^{(r)}\left(u_{l, x}, \frac{r}{\eta} c_{x}\right)\right]
\end{aligned}
$$

Taking into account (4.32) and (4.36), it is possible to write an expression for the total azimuthal stress. This expression reads

$$
\begin{aligned}
\left\langle 0\left|T_{2}^{2}\right| 0\right\rangle= & \frac{2^{1 / 2}}{\pi^{5 / 2} \alpha^{4}} \sum_{l=0}^{\infty} \cos (2 \pi \tilde{\imath})\left[\sum_{k=0}^{p}(-1)^{k} \cos \left(2 k \pi a_{0}\right) \cos (k \pi / q) \mathcal{M}_{l}^{(\phi)}\left(u_{l, k}, \frac{r}{\eta} s_{k}\right)\right. \\
& \left.+\frac{q}{\pi} \int_{0}^{\infty} d x \frac{\sinh (x) h\left(q, a_{0}, 2 x\right)}{\cosh (2 q x)-\cos (q \pi)} \mathcal{M}_{l}^{(\phi)}\left(u_{l, x}, \frac{r}{\eta} c_{x}\right)\right],
\end{aligned}
$$

where the contribution induced by the cosmic string is the $l=0$ term of the above equation with the coefficient $1 / 2$.

\section{Axial stress}

For the axial stress, considering the equation (4.1) after inserting the expression for the eigenfunctions, one finds

$$
\left\langle 0\left|T_{3}^{3}\right| 0\right\rangle=\frac{q \eta^{5}}{\alpha^{4} \pi^{2} L} \sum_{l=-\infty}^{\infty} \sum_{j} \int_{0}^{\infty} d p p\left[J_{\beta_{1}}^{2}(p r)+J_{\beta_{2}}^{2}(p r)\right] k_{l}^{2} \operatorname{Re}\left[K_{1 / 2-i m \alpha}(i \gamma \eta) K_{1 / 2-i m \alpha}(-i \gamma \eta)\right] .
$$

To solve the summation over $l$ we consider the Abel-Plana summation formula (3.6) with the functions $g(u)=k^{2}$ and $f(u)$ given by the real part of (3.8). For the contribution induced by the cosmic string and the de Sitter spacetime, using the first term of (3.6) and the representation (A3), after similar transformations done previously one finds

$$
\left\langle 0\left|T_{3}^{3}\right| 0\right\rangle_{s}^{d S}=\frac{q \alpha^{-4}}{2^{3 / 2} \pi^{5 / 2}} \int_{0}^{\infty} d z z^{3 / 2} e^{z\left(1-r^{2} / \eta^{2}\right)} \operatorname{Re}\left[K_{1 / 2-i m \alpha}(z)\right] \mathcal{J}\left(q, a_{0}, z r^{2} / \eta^{2}\right)
$$

where we can note that

$$
\left\langle 0\left|T_{3}^{3}\right| 0\right\rangle_{s}^{d S}=\left\langle 0\left|T_{0}^{0}\right| 0\right\rangle_{s}^{d S}
$$

The axial stress induced by the compactification is obtained from the second term in (3.6). By using this formula and taking into account similar transformations done previously for the contributions induced by the compactification we obtain

$$
\begin{aligned}
\left\langle 0\left|T_{3}^{3}\right| 0\right\rangle_{c}= & -\frac{q \eta^{5}}{\alpha^{4} \pi^{2}} \sum_{l=1}^{\infty} \cos (2 \pi l \tilde{\beta}) \sum_{j} \int_{0}^{\infty} d p p\left[J_{\beta_{1}}^{2}(p r)+J_{\beta_{2}}^{2}(p r)\right] \int_{p}^{\infty} d u u^{2} e^{-l L u} \\
& \times \operatorname{Re}\left\{K_{1 / 2-i m \alpha}(\eta \lambda)\left[I_{1 / 2-i m \alpha}(\eta \lambda)+I_{-1 / 2+i m \alpha}(\eta \lambda)\right]\right\},
\end{aligned}
$$


where again we have considered (3.28). Knowing $u^{2} e^{-l L u}=l^{-2} \partial_{L}^{2} e^{-l L u}$ and using the representation (3.30) we are able to solve the integrals over $p$ and $u$ which gives

$$
\begin{aligned}
\left\langle 0\left|T_{3}^{3}\right| 0\right\rangle_{c}= & \frac{q \eta^{5}}{4 \alpha^{4} \pi^{5 / 2}} \sum_{l=1}^{\infty} \cos (2 \pi i \tilde{\beta}) \int_{0}^{\infty} d s\left(1-\frac{l^{2} L^{2}}{2 s^{2}}\right) \frac{e^{-\left(l^{2} L^{2} / 2+r^{2}-\eta^{2}\right) /\left(2 s^{2}\right)}}{s^{6}} \\
& \times \operatorname{Re}\left[K_{1 / 2-i m \alpha}\left(\eta^{2} / 2 s^{2}\right)\right] \mathcal{J}\left(q, a_{0}, r^{2} / 2 s^{2}\right) .
\end{aligned}
$$

By making the change of variables $z=\eta^{2} / 2 s^{2}$ and also using the representation (3.14) we note that the following relation holds

$$
\left\langle 0\left|T_{3}^{3}\right| 0\right\rangle_{c}=\partial_{L}\left[L\left\langle 0\left|T_{1}^{1}\right| 0\right\rangle_{c}\right]
$$

Taking into the consideration the expression for the radial stress induced by the compactification, the axial stress is given by

$$
\begin{aligned}
\left\langle 0\left|T_{3}^{3}\right| 0\right\rangle_{c}= & \frac{2^{3 / 2}}{\pi^{5 / 2} \alpha^{4}} \sum_{l=1}^{\infty} \cos (2 \pi l \tilde{\beta})\left[\sum_{k=0}^{p}(-1)^{k} \cos \left(2 k \pi a_{0}\right) \cos (k \pi / q) \mathcal{M}_{l}^{(z)}\left(u_{l, k}, \frac{r}{\eta} s_{k}\right)\right. \\
& \left.+\frac{q}{\pi} \int_{0}^{\infty} d x \frac{\sinh (x) h\left(q, a_{0}, 2 x\right)}{\cosh (2 q x)-\cos (q \pi)} \mathcal{M}_{l}^{(z)}\left(u_{l, x}, \frac{r}{\eta} c_{x}\right)\right]
\end{aligned}
$$

with the notation

$$
\begin{aligned}
\mathcal{M}_{l}^{(z)}\left(u_{l, y}, v\right)= & \frac{\sqrt{\pi}}{2^{5 / 2}} \operatorname{Re}\left\{\frac { \Gamma ( 3 - i m \alpha ) \Gamma ( 2 + i m \alpha ) } { u _ { l , y } ^ { 6 - i m \alpha } } \left[u_{l, y}^{2}\left((2 i m \alpha-5) \frac{l^{2} L^{2}}{2 \eta^{2}}+2 v^{2}-1\right) F\left(a, b ; c ; d_{l, y}\right)\right.\right. \\
& \left.\left.+\frac{1}{3}(3-i m \alpha)(4-i m \alpha) \frac{l^{2} L^{2}}{2 \eta^{2}} F\left(a+1, b+1 ; c+1 ; d_{l, y}\right)\right]\right\} .
\end{aligned}
$$

\section{PROPERTIES OF THE VEV OF THE ENERGY-MOMENTUM TENSOR}

In this section, we plan to analyze some properties of the VEVs of the energy-momentum tensor. As we have shown, the VEVs of the energy-momentum tensor can be decomposed into a contribution induced by the curvature of the de Sitter spacetime and the cosmic string, and another one induced by the compactification. The first contribution, after removing the pure dS part, can be written in a compact form as (no summation over $\mu$ )

$$
\begin{array}{r}
\left\langle 0\left|T_{\mu}^{\mu}\right| 0\right\rangle_{s}=\frac{2^{1 / 2}}{\pi^{5 / 2} \alpha^{4}}\left[\sum_{k=1}^{p}(-1)^{k} \cos \left(2 k \pi a_{0}\right) \cos (k \pi / q) \mathcal{M}_{0}\left(u_{0, k}\right)\right. \\
\left.+\frac{q}{\pi} \int_{0}^{\infty} d x \frac{\sinh (x) h\left(q, a_{0}, 2 x\right)}{\cosh (2 q x)-\cos (q \pi)} \mathcal{M}_{0}\left(u_{0, x}\right)\right],
\end{array}
$$

for $\mu=t, r, z$ where the functions $\mathcal{M}_{0}\left(u_{0, y}\right)$ were defined in (4.13), and also (3.21)-(3.24). This contribution of the energymomentum tensor is an even function of $a_{0}$. Considering a massless fermionic field, the above expression is simplified to

$$
\begin{aligned}
\left\langle 0\left|T_{\mu}^{\mu}\right| 0\right\rangle_{s}= & \frac{1}{4 \pi^{2} \alpha^{4}}\left[\sum_{k=1}^{p}(-1)^{k} \frac{\cos \left(2 k \pi a_{0}\right) \cos (k \pi / q)}{u_{0, k}^{3}} F\left(2,3 / 2 ; 3 ; 1-u_{0, k}^{-2}\right)\right. \\
& \left.+\frac{q}{\pi} \int_{0}^{\infty} d x \frac{\sinh (x) h\left(q, a_{0}, 2 x\right)}{\cosh (2 q x)-\cos (q \pi)} u_{0, x}^{-3} F\left(2,3 / 2 ; 3 ; 1-u_{0, x}^{-2}\right)\right] .
\end{aligned}
$$

Now, let us consider some asymptotic behavior of the expression (5.1). Near the string, $r / \eta \ll 1$, we proceed in a similar way as was done in the string part of the FC. First, we write (5.1) as 


$$
\begin{aligned}
\left\langle 0\left|T_{\mu}^{\mu}\right| 0\right\rangle_{s}= & \frac{2^{1 / 2}}{\pi^{5 / 2} \alpha^{4}}\left[\sum_{k=1}^{p}(-1)^{k} \cos \left(2 k \pi a_{0}\right) \cos (k \pi / q) \int_{0}^{\infty} d z z^{3 / 2} e^{-z\left(2 r^{2} s_{k}^{2} / \eta^{2}-1\right)}\right. \\
& \left.+\frac{q}{\pi} \int_{0}^{\infty} d x \frac{\sinh (x) h\left(q, a_{0}, 2 x\right)}{\cosh (2 q x)-\cos (q \pi)} \int_{0}^{\infty} d z z^{3 / 2} e^{-z\left(2 r^{2} c_{x}^{2} / \eta^{2}-1\right)}\right] \operatorname{Re}\left[K_{1 / 2-i m \alpha}(z)\right] .
\end{aligned}
$$

For the region near the string, the main contribution to the integral over $z$ comes from the regions near the upper limit of the integration, and we can use the expression for the Macdonald function considering large arguments. To the leading order we obtain

$$
\left\langle 0\left|T_{\mu}^{\mu}\right| 0\right\rangle_{s} \approx \frac{1}{2 \pi^{2}}\left(\frac{\eta}{\alpha r}\right)^{4}\left[\sum_{k=1}^{p}(-1)^{k} \frac{\cos \left(2 k \pi a_{0}\right) \cos (k \pi / q)}{s_{k}^{4}}+\frac{q}{\pi} \int_{0}^{\infty} d x \frac{c_{x}^{-4} \sinh (x) h\left(q, a_{0}, 2 x\right)}{\cosh (2 q x)-\cos (q \pi)}\right],
$$

where we note a divergence with a fourth power of the proper distance.

On the other hand, for regions where $r / \eta \gg 1$, we have

$$
\mathcal{M}_{0}\left(u_{0, y}\right) \approx \operatorname{Re}\left[\frac{\Gamma(1 / 2-i m \alpha) \Gamma(2+i m \alpha)}{2^{2 i m \alpha+5 / 2}\left(u_{0, y}+1\right)^{2+i m \alpha}}\right],
$$

and to the leading order, Eq. (5.1) reads

$$
\begin{aligned}
\left\langle 0\left|T_{\mu}^{\mu}\right| 0\right\rangle_{s} \approx & \frac{2^{1 / 2}}{\pi^{5 / 2}}\left(\frac{\eta}{\alpha r}\right)^{4} \operatorname{Re}\left\{\frac { \Gamma ( 1 / 2 - i m \alpha ) \Gamma ( 2 + i m \alpha ) } { 2 ^ { 2 i m \alpha + 5 / 2 } } ( \frac { \eta } { r } ) ^ { 2 i m \alpha } \left[\sum_{k=1}^{p}(-1)^{k} \frac{\cos \left(2 k \pi a_{0}\right) \cos (k \pi / q)}{s_{k}^{4+2 i m \alpha}}\right.\right. \\
& \left.\left.+\frac{q}{\pi} \int_{0}^{\infty} d x \frac{\sinh (x) h\left(q, a_{0}, 2 x\right)}{\cosh (2 q x)-\cos (q \pi)} c_{x}^{-4-2 i m \alpha}\right]\right\} .
\end{aligned}
$$

In the Fig. 3 we show the behavior of the string part of the energy density as function of $r^{2} / \eta^{2}$ (left plot) and of $m \alpha$ (right plot). As in the case of the string part of FC, the behavior of the string part of the energy density is highly affected by the value of the azimuthal magnetic flux. The turning point is around $a_{0} \approx 0.23$ for the chosen parameters in the figure.

In a similar way, the contribution due to the compactification, can be written as (no summation over $\mu$ )

$$
\begin{aligned}
\left\langle 0\left|T_{\mu}^{\mu}\right| 0\right\rangle_{c}= & \frac{2^{3 / 2}}{\pi^{5 / 2} \alpha^{4}} \sum_{l=1}^{\infty} \cos (2 \pi l \tilde{\beta})\left[\sum_{k=0}^{p}(-1)^{k} \cos \left(2 k \pi a_{0}\right) \cos (k \pi / q) \mathcal{M}_{l}\left(u_{l, k}\right)\right. \\
& \left.+\frac{q}{\pi} \int_{0}^{\infty} d x \frac{\sinh (x) h\left(q, a_{0}, 2 x\right)}{\cosh (2 q x)-\cos (q \pi)} \mathcal{M}_{l}\left(u_{l, x}\right)\right]
\end{aligned}
$$

for $\mu=t, r$. The previous expression is an even function of the parameters $\tilde{\beta}$ and $a_{0}$. Considering $r / \eta \ll 1$, the leading term of the above expression reads
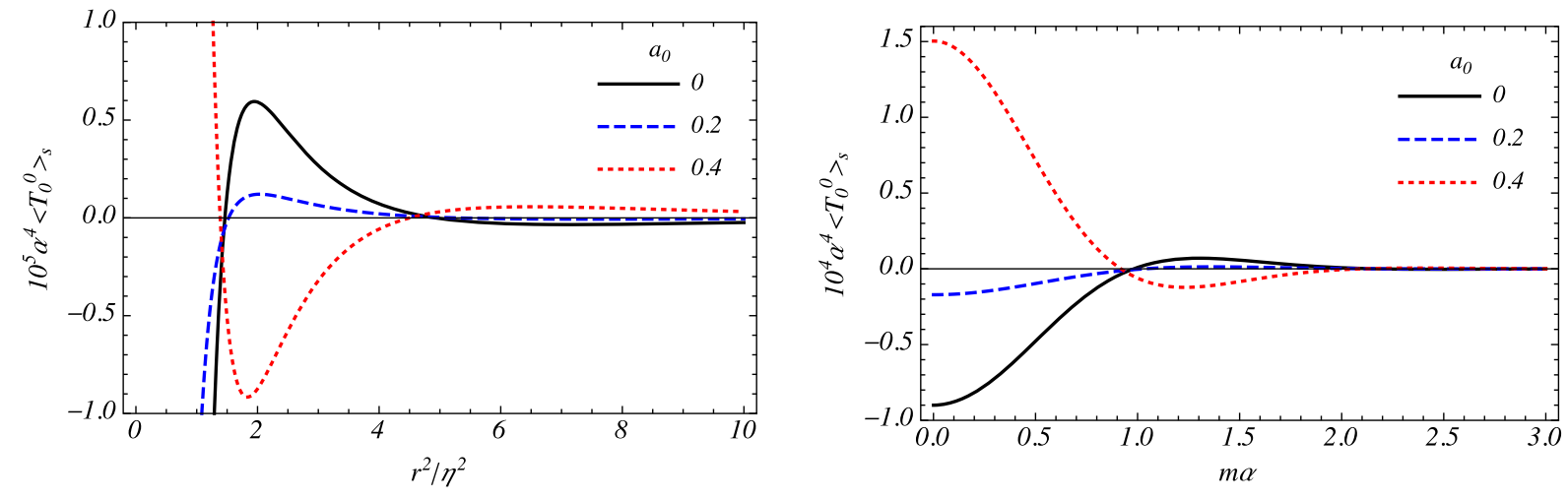

FIG. 3. Energy density and azimuthal stress induced by the magnetic flux and planar angle deficit as a function of $r^{2} / \eta^{2}$ (left plots) for $m \alpha=2$ and the mass of the field (right plots) considering $r / \eta=2$. In both plots $q=1.5$. 


$$
\begin{aligned}
\left\langle 0\left|T_{\mu}^{\mu}\right| 0\right\rangle_{c} \approx & \frac{q \cos \left[q \pi\left(1 / 2+\left|a_{0}\right|\right)\right] \Gamma\left(1 / 2-q\left(1 / 2-\left|a_{0}\right|\right)\right)}{\pi^{7 / 2} \alpha^{4}}\left(\frac{r}{\sqrt{2} \eta}\right)^{q\left(1-2\left|a_{0}\right|\right)-1} \\
& \times \sum_{l=1}^{\infty} \cos (2 \pi l \tilde{\beta}) \operatorname{Re}\left\{\frac{\Gamma(2+i m \alpha) \Gamma\left(3 / 2-i m \alpha+q\left(1-2\left|a_{0}\right|\right)\right)}{2^{2+i m \alpha} \Gamma(2-i m \alpha)\left(l L /(\sqrt{2} \eta)^{3-i m \alpha+q\left(1-2\left|a_{0}\right|\right)}\right)}\right\} .
\end{aligned}
$$

This expression is divergent if $\left|a_{0}\right|>(1-1 / q) / 2$ and is finite if $\left|a_{0}\right| \leq(1-1 / q) / 2$ in the limit $r=0$. For $a_{0}=0$ the above expression vanishes on the string's core.

The Eq. (5.7) can be decomposed as

$$
\left\langle 0\left|T_{\mu}^{\mu}\right| 0\right\rangle_{c}=\left\langle 0\left|T_{\mu}^{\mu}\right| 0\right\rangle_{c}^{(0)}+\left\langle 0\left|T_{\mu}^{\mu}\right| 0\right\rangle_{c}^{\left(q, a_{0}\right)}
$$

The first term is the $k=0$ contribution with the coefficient $1 / 2$ in (5.7). This is a pure topological term, independent of the radial coordinate, the azimuthal magnetic flux and the conical defect. This contribution is induced only by the compactification which is given by

$$
\left\langle 0\left|T_{\mu}^{\mu}\right| 0\right\rangle_{c}^{(0)}=\frac{2^{1 / 2}}{\pi^{5 / 2} \alpha^{4}} \sum_{l=1}^{\infty} \cos (2 \pi l \tilde{\beta}) \mathcal{M}_{l}\left(u_{l, 0}\right) .
$$

The second contribution on the right-hand side of (5.7) which depends on the planar angle deficit, magnetic flux and compactification, is given by

$$
\begin{aligned}
\left\langle 0\left|T_{\mu}^{\mu}\right| 0\right\rangle_{c}^{\left(q, a_{0}\right)}= & \frac{2^{3 / 2}}{\pi^{5 / 2} \alpha^{4}} \sum_{l=1}^{\infty} \cos (2 \pi l \tilde{\beta})\left[\sum_{k=1}^{p}(-1)^{k} \cos \left(2 k \pi a_{0}\right) \cos (k \pi / q) \mathcal{M}_{l}\left(u_{l, k}\right)\right. \\
& \left.+\frac{q}{\pi} \int_{0}^{\infty} d x \frac{\sinh (x) h\left(q, a_{0}, 2 x\right)}{\cosh (2 q x)-\cos (q \pi)} \mathcal{M}_{l}\left(u_{l, x}\right)\right] .
\end{aligned}
$$

For $L / \eta \gg 1$ and fixed $r / \eta$, to the leading order, we have

$$
\begin{aligned}
\left\langle 0\left|T_{\mu}^{\mu}\right| 0\right\rangle_{c}^{\left(q, a_{0}\right)} \approx & \frac{2^{3 / 2}}{\pi^{5 / 2}}\left(\frac{\eta}{\alpha L}\right)^{4} \sum_{l=1}^{\infty} \cos (2 \pi i \tilde{\beta}) \operatorname{Re}\left\{\frac{\Gamma(1 / 2-i m \alpha) \Gamma(2+i m \alpha)}{2^{i m \alpha+1 / 2}}\left(\frac{\eta}{L}\right)^{2 i m \alpha}\right. \\
& \left.\times\left[\sum_{k=1}^{p} \frac{(-1)^{k} \cos \left(2 k \pi a_{0}\right) \cos (k \pi / q)}{\left(\frac{l^{2}}{2}+\frac{2 r^{2} s_{k}^{2}}{L^{2}}\right)^{2+i m \alpha}}+\frac{q}{\pi} \int_{0}^{\infty} d x \frac{\sinh (x) h\left(q, a_{0}, 2 x\right)}{\cosh (2 q x)-\cos (q \pi)}\left(\frac{l^{2}}{2}+\frac{2 r^{2} c_{x}^{2}}{L^{2}}\right)^{-2-i m \alpha}\right]\right\} .
\end{aligned}
$$

In Fig. 4 we show the behavior of the energy density induced by the compactification as a function of $L / \eta$ (left plot) and $\tilde{\beta}$ (right plot). We note that, this contribution of the energy density goes to zero for large values of $L$, as expected.
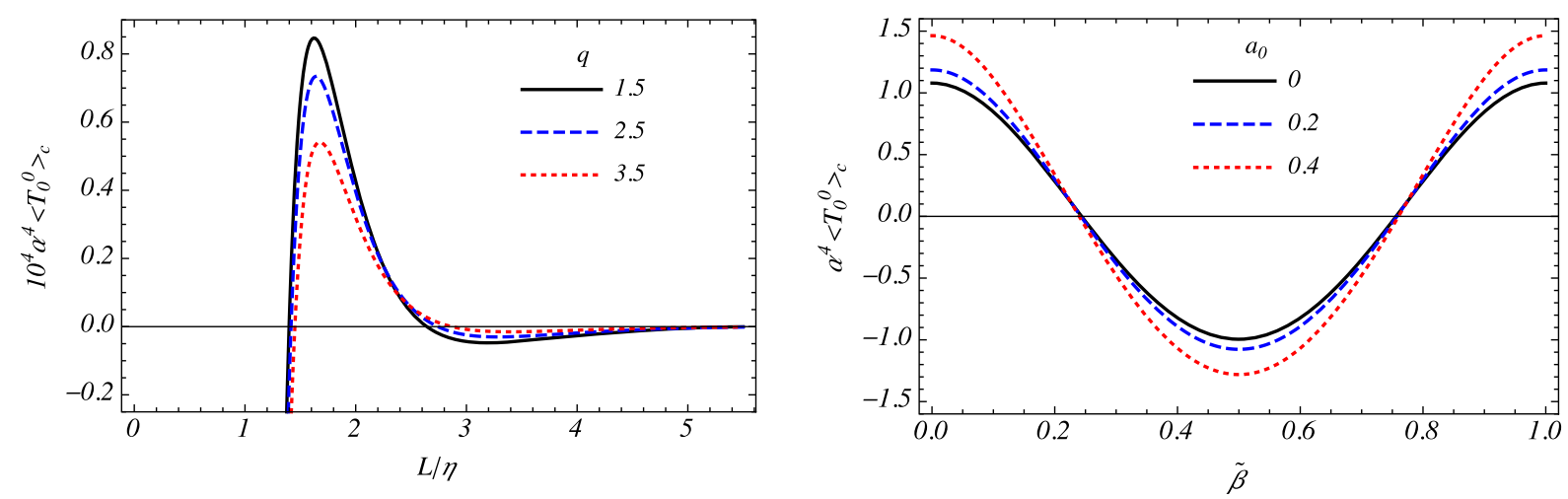

FIG. 4. Energy density induced by the compactification as a function of $L / \eta$ (left plot) for $\tilde{\beta}=r / \eta=a_{0}=0.25$, considering different values of $q$ and as a function of $\tilde{\beta}$ (right plot) for $L / \eta=0.75, r / \eta=0.25$ and $q=1.5$, for three different values of $a_{0}$. 
Now, let us evaluate some special cases of the azimuthal stress given by the Eq. (4.37). For a massless fermion field the azimuthal stress reads

$$
\begin{aligned}
\left\langle 0\left|T_{2}^{2}\right| 0\right\rangle= & \frac{1}{2 \pi^{2} \alpha^{4}} \sum_{l=0}^{\infty} \cos (2 \pi l \tilde{\beta})\left[\sum_{k=0}^{p}(-1)^{k} \cos \left(2 k \pi \alpha_{0}\right) \cos (k \pi / q) \mathcal{B}_{l}\left(u_{l, k}, \frac{r}{\eta} s_{k}\right)\right. \\
& \left.+\frac{q}{\pi} \int_{0}^{\infty} d x \frac{h\left(q, a_{0}, 2 x\right) \sinh x}{\cosh (2 q x)-\cos (q \pi)} \mathcal{B}_{l}\left(u_{l, x}, \frac{r}{\eta} c_{x}\right)\right],
\end{aligned}
$$

with the notation

$$
\mathcal{B}_{l}^{(\phi)}\left(u_{l, y}, v\right)=\frac{\left(\frac{l^{2} L^{2}}{2 \eta^{2}}-10 v^{2}-1\right)}{u_{l, y}^{4}} F\left(2,3 / 2 ; 3 ; 1-u_{l, y}^{-2}\right)+\frac{4 v^{2}}{u_{l, y}^{6}} F\left(3,5 / 2 ; 4 ; 1-u_{l, y}^{-2}\right)
$$

In the absence of the magnetic flux in the azimuthal direction, $a_{0}=0$, we have

$$
\begin{aligned}
\left\langle 0\left|T_{2}^{2}\right| 0\right\rangle= & \frac{2^{1 / 2}}{\pi^{5 / 2} \alpha^{4}} \sum_{l=0}^{\infty} \cos (2 \pi l \tilde{\beta})\left[\sum_{k=0}^{p}(-1)^{k} \cos (k \pi / q) \mathcal{M}_{l}^{(\phi)}\left(u_{l, k}, \frac{r}{\eta} s_{k}\right)\right. \\
& \left.+\frac{2 q \cos (q \pi / 2)}{\pi} \int_{0}^{\infty} d x \frac{\sinh (x) \sinh (q x)}{\cosh (2 q x)-\cos (q \pi)} \mathcal{M}_{l}^{(\phi)}\left(u_{l, x}, \frac{r}{\eta} c_{x}\right)\right] .
\end{aligned}
$$

The axial stress induced by the compactification is given by the Eq. (4.44). Considering a massless fermion field, this expression reads

$$
\begin{aligned}
\left\langle 0\left|T_{3}^{3}\right| 0\right\rangle_{c}= & \frac{1}{\pi^{2} \alpha^{4}} \sum_{l=1}^{\infty} \cos (2 \pi l \tilde{\beta})\left[\sum_{k=0}^{p}(-1)^{k} \cos \left(2 k \pi a_{0}\right) \cos (k \pi / q) \mathcal{B}_{l}^{(z)}\left(u_{l, k}, \frac{r}{\eta} s_{k}\right)\right. \\
& \left.+\frac{q}{\pi} \int_{0}^{\infty} d x \frac{\sinh (x) h\left(q, a_{0}, 2 x\right)}{\cosh (2 q x)-\cos (q \pi)} \mathcal{B}_{l}^{(z)}\left(u_{l, x}, \frac{r}{\eta} c_{x}\right)\right],
\end{aligned}
$$

with the notation

$$
\mathcal{B}_{l}^{(z)}\left(u_{l, y}, v\right)=\frac{\left(2 v^{2}-\frac{5 l^{2} L^{2}}{2 \eta^{2}}-1\right)}{u_{l, y}^{4}} F\left(2,3 / 2 ; 3,1-u_{l, y}^{-2}\right)-\frac{2 l^{2} L^{2} / \eta^{2}}{u_{l, y}^{6}} F\left(3,5 / 2 ; 4,1-u_{l, y}^{-2}\right) .
$$

Again, it is easy to see that the axial stress induced by the compactification can be decomposed as

$$
\left\langle 0\left|T_{3}^{3}\right| 0\right\rangle_{c}=\left\langle 0\left|T_{3}^{3}\right| 0\right\rangle_{c}^{(0)}+\left\langle 0\left|T_{3}^{3}\right| 0\right\rangle_{c}^{\left(q, a_{0}\right)}
$$

The first contribution of the above decomposition is the axial stress induced only by the compactification, being a pure topological term independent of the radial coordinate and the magnetic flux in the azimuthal direction. This part is the $k=0$ term in (4.44) which is given by

$$
\left\langle 0\left|T_{3}^{3}\right| 0\right\rangle_{c}^{(0)}=\frac{2^{1 / 2}}{\pi^{5 / 2} \alpha^{4}} \sum_{l=1}^{\infty} \cos (2 \pi l \tilde{\beta}) \mathcal{M}_{l}^{(z)}\left(u_{l, 0}, \frac{r}{\eta} s_{0}\right) .
$$

The second term on the right-hand side of (5.18), the compactification contribution to the axial stress dependent on the planar angle deficit and the magnetic flux, has the form

$$
\begin{aligned}
\left\langle 0\left|T_{3}^{3}\right| 0\right\rangle_{c}= & \frac{2^{3 / 2}}{\pi^{5 / 2} \alpha^{4}} \sum_{l=1}^{\infty} \cos (2 \pi l \tilde{\beta})\left[\sum_{k=1}^{p}(-1)^{k} \cos \left(2 k \pi a_{0}\right) \cos (k \pi / q) \mathcal{M}_{l}^{(z)}\left(u_{l, k}, \frac{r}{\eta} s_{k}\right)\right. \\
& \left.+\frac{q}{\pi} \int_{0}^{\infty} d x \frac{\sinh (x) h\left(q, a_{0}, 2 x\right)}{\cosh (2 q x)-\cos (q \pi)} \mathcal{M}_{l}^{(z)}\left(u_{l, x}, \frac{r}{\eta} c_{x}\right)\right],
\end{aligned}
$$

where we note that the axial stress induced by the compactification is an even function of the parameters $\tilde{\beta}$ and $a_{0}$. 
Two important relations obeyed by the fermionic energymomentum tensor are the conservation condition and the trace relation. The latter can be obtained by using the general expression for the energy-momentum tensor, Eq. (4.1), combined with the equation of motion. We can start with the trace relation. Because the contribution to the energy-momentum tensor associated with the cosmic string and compactification present no anomalies, the trace relations read

$$
\left\langle 0\left|T_{\mu}^{\mu}\right| 0\right\rangle_{s}=m\langle\bar{\psi} \psi\rangle_{s}, \quad\left\langle 0\left|T_{\mu}^{\mu}\right| 0\right\rangle_{c}=m\langle\bar{\psi} \psi\rangle_{c},
$$

where we observe that for a massless field the VEV of the energy-momentum tensor is traceless. As to the conservation condition, $\nabla_{\alpha}\left\langle 0\left|T_{\beta}^{\alpha}\right| 0\right\rangle=0$, in the configuration that we are studying here, this equation is reduced only to one differential equation

$$
\partial_{r}\left[r\left\langle 0\left|T_{1}^{1}\right| 0\right\rangle\right]=\left\langle 0\left|T_{2}^{2}\right| 0\right\rangle .
$$

Note that we have already proved this equation during the evaluation of the energy-momentum tensor in the previous sections.

\section{CONCLUSION}

In the present paper, we have studied the influence of the combined effects of the spacetime background and the topology on the FC and the VEV of the energy-momentum tensor associated with a massive spinor field. As the background geometry we have considered the dS spacetime, maximally symmetric curved space, conformally flat with constant positive curvature, which plays an important role in the quantum field theory and most importantly in cosmology. We have investigated the effect of the presence of the cosmic string in the aforementioned spacetime and a magnetic flux as well as the compactification of the spatial dimension along the string inducing topological effects in a fermionic system, considering that the fermion field obeys a quasiperiodic condition (2.4).

The FC has been evaluated by using the direct summation over the fermionic modes, prepared in the BunchDavies vacuum state, given by (2.6). The momentum along the string direction becomes discrete due the compactification along this direction and in order to evaluate the summation over this quantum number, we have employed the Abel-Plana summation formula (3.6). Consequently, both the FC and the VEV of the energy-momentum tensor are decomposed into a contribution induced by a cosmic string in the $\mathrm{dS}$ space with no compactification, and another one induced by the compactification.

The string part of the FC is given by (3.20), where this contribution is an even function of the magnetic flux along the azimuthal direction and depends on the ratio between the radial and conformal time coordinates, $r / \eta$, which is the proper distance from the string measured in units of the $\mathrm{dS}$ curvature scale $\alpha$. Some particular cases of the FC contribution induced by the cosmic string in $\mathrm{dS}$ spacetime have been considered. For points near the string the FC is given by (3.25) where the leading term presents a divergence with the second power of the proper distance and for large distances from the string the leading term of the $\mathrm{FC}$ is given by (3.26). In Fig. 1 we show the influence of the magnetic flux in the azimuthal direction on the FC as function of $r^{2} / \eta^{2}$ and $m \alpha$. The FC induced by the compactification is given by (3.32) being an even function of the magnetic flux in the azimuthal and axial directions. This induced contribution of the FC can be decomposed into two parts as shown in (3.33). The first part, given by (3.34) is a pure topological term induced only by the compactification and independent of the parameters $q, a_{0}$ and the radial coordinate. The second part, Eq. (3.35), is the contribution of the FC induced by the compactification, the planar angle deficit and the magnetic fluxes. For regions near the string, the leading term of the FC induced by the compactification is given by (3.36), which is finite on the string if $\left|a_{0}\right|=(1-1 / q) / 2$, vanishes for $\left|a_{0}\right|<(1-1 / q) / 2$ and diverges if $\left|a_{0}\right|>(1-1 / q) / 2$. We have also studied the limit of large values of the compactification, $L / \eta \gg 1$, where the leading term is given by Eq. (3.37). Figure 2 shows the behavior of the FC induced by the compactification as a function of $L / \eta$ and the axial magnetic flux. We have shown that both induced parts of the FC vanish for a massless fermion field.

Another important quantity that characterizes the fermionic vacuum is the VEV of the energy-momentum tensor. The steps of the evaluation of each component of this tensor has been presented in Sec. IV while in Sec. V we have presented combined expressions for both cosmic string in dS spacetime without compactification and topological contribution of the energy-momentum tensor. The $\mathrm{VEV}$ of the energy-momentum tensor in dS space in the presence of a cosmic string is given in a compact form (5.1) which includes the energy density, the radial and the axial stresses. This contribution is an even function of the azimuthal magnetic flux. Some special cases of this expression have been evaluated. For a massless fermionic field we have the expression (5.2). Considering $r / \eta \ll 1$, there is a divergence with a fourth power of the proper distance, (5.4). On the other hand, for large distances from the string, this contribution goes to zero as one can see in the Eq. (5.6). In Fig. 3 we have plotted the effect of the parameter $a_{0}$ on the energy-density as a function of $r^{2} / \eta^{2}$ and $m \alpha$. For the contribution induced by the compactification, the energy density and the radial stress are written in the compact form (5.7). They are even functions of the magnetic fluxes in azimuthal and axial directions. For regions near the string, this part of the energy-momentum tensor is given by (5.8) being divergent on the string if $\left|a_{0}\right|>(1-1 / q) / 2$ and finite if $\left|a_{0}\right| \leq(1-1 / q) / 2$. 
We have also decomposed this part of the energy-momentum tensor into two parts: the first one is purely topological, (5.10), independent of the planar angle deficit, the azimuthal magnetic flux and the radial coordinate, while the second one, (5.11), is dependent on the compactification and other parameters that characterize the system under consideration. In the regime where $L / \eta \gg 1$, the latter has been given by (5.12). In Fig. 4 we have plotted the behavior of the energy density as a function of $L / \eta$ and $\tilde{\beta}$.

The azimuthal and axial stresses induced by the compactification have been evaluated in the Secs. IV C and IV D, respectively. An expression for the total azimuthal stress and the axial stress induced by the compactification has been written in compact forms given by (4.37) and (4.44), respectively. Some particular cases of these components of the energy-momentum tensor are presented in the Sec. V. Moreover, for a massless spinor field in the absence of the azimuthal flux, $a_{0}=0$, the azimuthal stress is given by (5.13) and (5.15), respectively. The axial stress induced by the compactification considering a massless fermionic field is given by (5.16). This induced part of the axial stress has been decomposed into two parts: a pure topological part, (5.19), independent of the parameters $q, a_{0}$ and the radial coordinate, and a part dependent on these parameters, (5.20), where the latter is an even function of both magnetic fluxes.

We have also verified that the components of the energymomentum tensor obey the trace relation (5.21) and the energy-momentum conservation in the covariant form. In particular, for a massless fermion field the energymomentum tensor is traceless.

\section{ACKNOWLEDGMENTS}

E. A. F. B and A. M. would like to thank the Brazilian agency CAPES for financial support. A. M. also thanks financial support from the Brazilian agency $\mathrm{CNPq}$ and Universidade Federal de Pernambuco Edital Qualis A.

\section{APPENDIX: RELEVANT RELATIONS FOR BESSEL, HANKEL, AND MACDONALD FUNCTIONS}

In this paper we use the following relations [49,54-56]

$$
\begin{gathered}
H_{\nu}^{(2)}(x)=\frac{2 i}{\pi} e^{i \pi \nu / 2} K_{\nu}(i x) \\
\left|H_{1 / 2-i m \alpha}^{(2)}(\gamma \eta)\right|^{2}=\frac{4 e^{\pi m \alpha}}{\pi^{2}}\left|K_{1 / 2-i m \alpha}(i \gamma \eta)\right|^{2} \\
\left|H_{-1 / 2-i m \alpha}^{(2)}(\gamma \eta)\right|^{2}=\frac{4 e^{\pi m \alpha}}{\pi^{2}}\left|K_{1 / 2+i m \alpha}(i \gamma \eta)\right|^{2} \\
K_{\nu}(x)=K_{-\nu}(x) \\
\left|K_{1 / 2-i m \alpha}(i x)\right|^{2}-\left|K_{1 / 2+i m \alpha}(i x)\right|^{2} \\
=-i\left(\partial_{x}+\frac{1-2 i m \alpha}{x}\right) K_{1 / 2-i m \alpha}(i x) K_{1 / 2-i m \alpha}(-i x)
\end{gathered}
$$

$$
\begin{aligned}
& K_{\nu}(i x) K_{\nu}(-i x) \\
& =\int_{0}^{\infty} d u u^{-1} \int_{0}^{\infty} d y \cosh (2 \nu y) \exp \left[-2 u x^{2} \sinh ^{2} y-1 /(2 u)\right]
\end{aligned}
$$$$
\left(u \partial_{u}+2 \nu\right) e^{u^{2}} K_{\nu}\left(u^{2}\right)=2 u^{2} e^{u^{2}}\left[K_{\nu}\left(u^{2}\right)-K_{\nu-1}\left(u^{2}\right)\right]
$$$$
\int_{0}^{\infty} d x x^{\alpha-1} e^{-p x} K_{\nu}(c x)=\frac{\Gamma(\alpha-\nu) e^{-i \pi \nu}}{\left(p^{2}+c^{2}\right)^{\alpha / 2}} Q_{\alpha-1}^{\nu}\left(\frac{p}{\sqrt{p^{2}-c^{2}}}\right)
$$

$$
\begin{aligned}
Q_{\nu}^{\mu}(z)= & \frac{e^{i \mu \pi} \sqrt{\pi} \Gamma(\nu+\mu+1)\left(z^{2}-1\right)^{\mu / 2}}{2^{\nu+1} \Gamma(\nu+3 / 2) z^{\nu+\mu+1}} \\
& \times F\left(1+\frac{\nu+\mu}{2}, \frac{\nu+\mu+1}{2} ; \nu+\frac{3}{2} ; z^{-2}\right)
\end{aligned}
$$

$$
\begin{aligned}
& F(a, b ; c ; z)= \frac{\Gamma(c) \Gamma(c-a-b)}{\Gamma(c-a) \Gamma(c-b)} F(a, b ; a+b-c ; 1-z) \\
&+\frac{\Gamma(c) \Gamma(a+b-c)}{(1-z)^{a+b-c} \Gamma(a) \Gamma(b)} \\
& \times F(c-a, c-b ; c-a-b+1 ; 1-z) \\
& K_{\nu}\left(e^{i m \pi} x\right)=e^{-i m \nu \pi} K_{\nu}(x)-i \pi \frac{\sin (m \nu \pi)}{\sin (\nu \pi)} I_{\nu}(x) \\
& K_{\nu}\left(e^{i \pi} x\right)-K_{\nu}\left(e^{-i \pi} x\right)=-i \pi\left[I_{\nu}(x)+I_{-\nu}(x)\right] \\
& \int_{0}^{\infty} d p p e^{-a p^{2}} J_{\nu}^{2}(p r)=\frac{e^{-r^{2} / 2 a}}{2 a} I_{\nu}\left(r^{2} / 2 a\right) \\
& \epsilon_{j}\left[J_{\beta_{1}}(x) J_{\beta_{2}}^{\prime}(x)-J_{\beta_{1}}^{\prime}(x) J_{\beta_{2}}(x)\right] \\
&=J_{\beta_{1}}^{2}(x)+J_{\beta_{2}}^{2}(x)-\frac{2 \beta_{1}+\epsilon_{j}}{x} J_{\beta_{1}}(x) J_{\beta_{2}}(x)
\end{aligned}
$$

$$
\begin{aligned}
& J_{\beta_{1}}(p r) J_{\beta_{2}}(p r)=\frac{1}{2 p}\left(\frac{2 \beta_{1}}{r}-\epsilon_{j} \partial_{r}\right) J_{\beta 1}^{2}(p r) \\
& \left(\epsilon_{j} \beta_{1}-r \partial_{r} / 2\right) e^{-r^{2} \tau} I_{\beta_{1}}\left(r^{2} \tau\right)=z e^{-z}\left[I_{\beta_{1}}(z)-I_{\beta_{2}}(z)\right]_{z=r^{2} \tau}
\end{aligned}
$$$$
\int_{0}^{\infty} d p p^{2} J_{\beta_{1}}(p r) J_{\beta_{2}}(p r) e^{-2 u \eta^{2} p^{2} \sinh ^{2} y}
$$$$
=\frac{2 \epsilon_{j}}{r} x e^{-x}\left[I_{\beta_{1}}(x)-I_{\beta_{2}}(x)\right]
$$$$
\left(2 \epsilon_{j} \beta_{1}+1\right)\left[I_{\beta_{1}}(x)-I_{\beta_{2}}(x)\right]
$$$$
=2\left(x \partial_{x}-x+1 / 2\right)\left[I_{\beta_{1}}(x)+I_{\beta_{2}}(x)\right]
$$$$
\epsilon_{j} J_{\beta_{1}}(x) J_{\beta_{2}}(x)=\frac{1}{x}\left(\epsilon_{j} \beta_{1}-\frac{x}{2} \partial_{x}\right) J_{\beta_{1}}^{2}(p r) \text {. }
$$$$
\left(\frac{\epsilon_{j} \beta_{1}}{x}-\partial_{x}\right) e^{-x} I_{\beta_{1}}(x)=e^{-x}\left[I_{\beta_{1}}(x)-I_{\beta_{x}}(x)\right]
$$ 
[1] A. Vilenkin and E. P. S. Shellard, Cosmic Strings and other Topological Defects, Cambridge Monographs on Mathematical Physics (Cambridge University Press, Cambridge, England, 1994).

[2] T. Damour and A. Vilenkin, Gravitational Wave Bursts from Cosmic Strings, Phys. Rev. Lett. 85, 3761 (2000).

[3] P. Bhattacharjee and G. Sigl, Origin and propagation of extremely high-energy cosmic rays, Phys. Rep. 327, 109 (2000).

[4] V. Berezinsky, B. Hnatyk, and A. Vilenkin, Gamma ray bursts from superconducting cosmic strings, Phys. Rev. D 64, 043004 (2001).

[5] S. Sarangi and S.-H. H. Tye, Cosmic string production towards the end of brane inflation, Phys. Lett. B 536, 185 (2002).

[6] E. J. Copeland, R. C. Myers, and J. Polchinski, Cosmic f-and d-strings, J. High Energy Phys. 06 (2004) 013.

[7] G. Dvali and A. Vilenkin, Formation and evolution of cosmic d strings, J. Cosmol. Astropart. Phys. 03 (2004) 010 .

[8] D. R. Nelson, Defects and Geometry in Condensed Matter Physics (Cambridge University Press, Cambridge, England, 2002).

[9] G. E. Volovik, The Universe in a Helium Droplet (Oxford University Press on Demand, Oxford, 2003), Vol. 117.

[10] H. B. Nielsen and P. Olesen, Vortex line models for dual strings, Nucl. Phys. B61, 45 (1973).

[11] D. Garfinkle, General relativistic strings, Phys. Rev. D 32, 1323 (1985).

[12] B. Linet, A vortex line model for infinite straight cosmic strings, Phys. Lett. A 124, 240 (1987).

[13] A. Linde, Inflationary cosmology, in Inflationary Cosmology (Springer, New York, 2008), pp. 1-54.

[14] B. Linet, Quantum field theory in the space-time of a cosmic string, Phys. Rev. D 35, 536 (1987).

[15] B. Allen and E. P. S. Shellard, On the evolution of cosmic strings, in The Formation and Evolution of Cosmic Strings: Proceedings of a Workshop Supported by the SERC and held in Cambridge, 1989, edited by G. W. Gibbons, S. W. Hawking, and T. Vachaspati (Cambridge University Press, Cambridge, England, 1990), pp. 421-448.

[16] M. Guimarães and B. Linet, Selfinteraction and quantum effects near a point mass in three-dimensional gravitation, Classical Quantum Gravity 10, 1665 (1993).

[17] P. Davies and V. Sahni, Quantum gravitational effects near cosmic strings, Classical Quantum Gravity 5, 1 (1988).

[18] V. P. Frolov and E. Serebryanyi, Vacuum polarization in the gravitational field of a cosmic string, Phys. Rev. D 35, 3779 (1987).

[19] B. Linet, Euclidean spinor Green's functions in the spacetime of a straight cosmic string, J. Math. Phys. (N.Y.) 36, 3694 (1995).

[20] E. S. Moreira, Jr., Massive quantum fields in a conical background, Nucl. Phys. B451, 365 (1995).

[21] V. B. Bezerra and N. R. Khusnutdinov, Vacuum expectation value of the spinor massive field in the cosmic string spacetime, Classical Quantum Gravity 23, 3449 (2006).

[22] J. Dowker, Vacuum averages for arbitrary spin around a cosmic string, Phys. Rev. D 36, 3742 (1987).
[23] M. Guimarães and B. Linet, Scalar Green's functions in an Euclidean space with a conical-type line singularity, Commun. Math. Phys. 165, 297 (1994).

[24] J. Spinelly and E. R. Bezerra de Mello, Vacuum polarization of a charged massless scalar field on cosmic string spacetime in the presence of a magnetic field, Classical Quantum Gravity 20, 873 (2003).

[25] J. Spinelly and E. R. Bezerra de Mello, Vacuum polarization by a magnetic field in the cosmic string space-time, Int. J. Mod. Phys. A 17, 4375 (2002).

[26] J. Spinelly and E. R. Bezerra de Mello, Vacuum polarization of a charged massless fermionic field by a magnetic flux in the cosmic string space-time, Int. J. Mod. Phys. D 13, 607 (2004).

[27] L. Sriramkumar, Fluctuations in the current and energy densities around a magnetic flux carrying cosmic string, Classical Quantum Gravity 18, 1015 (2001).

[28] Y. Sitenko and N. Vlasii, Induced vacuum current and magnetic field in the background of a cosmic string, Classical Quantum Gravity 26, 195009 (2009).

[29] E. R. Bezerra de Mello, Induced fermionic current densities by magnetic flux in higher dimensional cosmic string spacetime, Classical Quantum Gravity 27, 095017 (2010).

[30] E. R. Bezerra de Mello, V. Bezerra, A. Saharian, and V. Bardeghyan, Fermionic current densities induced by magnetic flux in a conical space with a circular boundary, Phys. Rev. D 82, 085033 (2010).

[31] E. R. Bezerra de Mello, V. B. Bezerra, A. A. Saharian, and H. H. Harutyunyan, Vacuum currents induced by a magnetic flux around a cosmic string with finite core, Phys. Rev. D 91, 064034 (2015).

[32] M. M. de Sousa, R. Ribeiro, and E. R. Bezerra de Mello, Induced fermionic current by a magnetic tube in the cosmic string spacetime, Phys. Rev. D 93, 043545 (2016).

[33] M. M. de Sousa, R. F. Ribeiro, and E. R. Bezerra de Mello, Fermionic vacuum polarization by an abelian magnetic tube in the cosmic string spacetime, Phys. Rev. D 95, 045005 (2017).

[34] A. H. Castro Neto, F. Guinea, N. M. R. Peres, K. S. Novoselov, and A. K. Geim, The electronic properties of graphene, Rev. Mod. Phys. 81, 109 (2009).

[35] S. Bellucci, A. Saharian, and V. Bardeghyan, Induced fermionic current in toroidally compactified spacetimes with applications to cylindrical and toroidal nanotubes, Phys. Rev. D 82, 065011 (2010).

[36] S. Bellucci and A. Saharian, Fermionic current from topology and boundaries with applications to higherdimensional models and nanophysics, Phys. Rev. D 87, 025005 (2013).

[37] E. R. Bezerra de Mello and A. Saharian, Fermionic current induced by magnetic flux in compactified cosmic string spacetime, Eur. Phys. J. C 73, 2532 (2013).

[38] S. Bellucci, E. R. Bezerra de Mello, A. de Padua, and A. Saharian, Fermionic vacuum polarization in compactified cosmic string spacetime, Eur. Phys. J. C 74, 2688 (2014).

[39] E. A. F. Bragança, H. F. S. Mota, and E. R. Bezerra de Mello, Induced vacuum bosonic current by magnetic flux in a higher dimensional compactified cosmic string spacetime, Int. J. Mod. Phys. D 24, 1550055 (2015). 
[40] E. A. F. Bragança, H. F. Santana Mota, and E. R. Bezerra de Mello, Vacuum expectation value of the energy-momentum tensor in a higher dimensional compactified cosmic string spacetime, Eur. Phys. J. Plus 134, 400 (2019).

[41] A. C. Ottewill and P. Taylor, Vacuum polarization on the schwarzschild metric threaded by a cosmic string, Phys. Rev. D 82, 104013 (2010).

[42] A. C. Ottewill and P. Taylor, Renormalized vacuum polarization and stress tensor on the horizon of a schwarzschild black hole threaded by a cosmic string, Classical Quantum Gravity 28, 015007 (2011).

[43] E. R. Bezerra de Mello and A. A. Saharian, Vacuum polarization by a cosmic string in de Sitter spacetime, J. High Energy Phys. 04 (2009) 046.

[44] E. R. Bezerra de Mello and A. A. Saharian, Fermionic vacuum polarization by a cosmic string in de Sitter spacetime, J. High Energy Phys. 08 (2010) 038.

[45] A. Mohammadi, E. R. Bezerra de Mello, and A. Saharian, Induced fermionic currents in de sitter spacetime in the presence of a compactified cosmic string, Classical Quantum Gravity 32, 135002 (2015).

[46] E. R. Bezerra de Mello and A. Saharian, Vacuum polarization induced by a cosmic string in anti-de sitter spacetime, J. Phys. A 45, 115402 (2012).

[47] E. R. Bezerra de Mello, E. F. Medeiros, and A. Saharian, Fermionic vacuum polarization by a cosmic string in anti-de sitter spacetime, Classical Quantum Gravity 30, 175001 (2013).
[48] W. O. dos Santos, H. F. Mota, and E. R. Bezerra de Mello, Induced current in high-dimensional AdS spacetime in the presence of a cosmic string and a compactified extra dimension, Phys. Rev. D 99, 045005 (2019).

[49] M. Abramowitz and I. Stegun, Handbook of Mathematical Functions (National Bureau of Standards, Washington DC, 1964).

[50] A. A. Saharian, The generalized Abel-Plana formula with applications to Bessel functions and Casimir effect, arXiv: 0708.1187.

[51] E. R. Bezerra de Mello and A. A. Saharian, Fermionic vacuum polarization by a composite topological defect in higher-dimensional space-time, Phys. Rev. D 78, 045021 (2008).

[52] S. Bellucci and A. A. Saharian, Fermionic casimir densities in toroidally compactified spacetimes with applications to nanotubes, Phys. Rev. D 79, 085019 (2009).

[53] S. Bellucci, E. R. Bezerra de Mello, and A. A. Saharian, Fermionic condensate in a conical space with a circular boundary and magnetic flux, Phys. Rev. D 83, 085017 (2011).

[54] G. N. Watson, A Treatise on the Theory of Bessel Functions (Cambridge University Press, Cambridge, England, 1995).

[55] I. S. Gradshtein and I. M. Ryzhik, Table of Integrals, Series, and Products (Academic Press, New York, 1980).

[56] A. Prudnikov, Y. Brychkov, and O. Marichev, Integrals and Series: Elementary Functions (Gordon and Breach Science Publishers, New York, 1986), Vol. 2. 